\title{
Dielectric friction, violation of the Stokes-Einstein-Debye relation, and non-Gaussian transport dynamics of dipolar solutes in water
}

\author{
Tuhin Samanta $\odot$ and Dmitry V. Matyushov ${ }^{*} *$ \\ Department of Physics and School of Molecular Sciences, Arizona State University, \\ P. O. Box 871604, Tempe, Arizona 85287-1604, USA
}

(Received 4 January 2021; accepted 19 March 2021; published 8 April 2021)

\begin{abstract}
The phenomenon of dielectric friction predicts slowing down of rotational and translational diffusion of a dipolar tracer in a polar medium due to retarded response of the medium polarization. This problem is studied here by numerical simulations in which the dipole moment of the tracer (solute) is continuously increased. The rotational time of the solute increases linearly with its squared dipole moment. A more pronounced effect of the solute dipole is found on the relaxation time of the electric field of the medium acting on the dipole: We report two orders of magnitude retardation for the electric field dynamics. With this strong retardation, classical theories of dielectric friction fail to describe slowing down of rotational diffusion. This failure is traced back to the breakdown of additivity between friction produced by van der Waals and electrostatic forces and torques. In contrast to the neglect of their correlations by traditional models, electrostatic and van der Waals forces and torques are strongly correlated. Electrostatic interactions bring to linear transport coefficients a number of features typically associated with the dynamics of low-temperature and supercooled liquids. The translational diffusion coefficient becomes strongly anisotropic in the solute's body frame, which translates to non-Gaussian translational dynamics. The Stokes-Einstein-Debye relation connecting the translational and rotational single-particle dynamics deviates from the hydrodynamic limit in linear proportion to the squared solute dipole. It is also found to increase with lowering temperature in qualitative agreement with phenomenology of supercooled liquids.
\end{abstract}

DOI: 10.1103/PhysRevResearch.3.023025

\section{INTRODUCTION}

Following the classical work by Einstein [1], mobility of a colloidal particle in a liquid has been viewed as a result of random collisions of liquid's molecules with the tagged particle [2-4]. While in principle correct, this view is far from accurate when applied to transport coefficients in liquids. Collisions in dense liquids never happen as single-particle events $[5,6]$, and it is more productive to think of collective liquid excitations leading to imbalances in forces acting on different sides of a tagged particle and producing the overall Browninan motion. For instance, translational friction is determined by statistics and dynamics of forces acting from the solvent on the tagged particle (solute). If the total fluctuating force from the solvent on the solute is $\mathbf{F}(t)$ and its fluctuation is $\delta \mathbf{F}(t)$, then the translational diffusion coefficient is given by the time integral of the force-force correlation function $[7,8]$

$$
D_{t}=\left[\left(\beta^{2} / 3\right) \int_{0}^{\infty} d t\langle\delta \mathbf{F}(t) \cdot \delta \mathbf{F}(0)\rangle\right]^{-1},
$$

\footnotetext{
*dmitrym@asu.edu
}

Published by the American Physical Society under the terms of the Creative Commons Attribution 4.0 International license. Further distribution of this work must maintain attribution to the author(s) and the published article's title, journal citation, and DOI. where $\beta=\left(k_{B} T\right)^{-1}$ is the inverse temperature. Equation (1) assumes Langevin dynamics without memory effects [5,9], as is commonly adopted for diffusion of a large solute.

The perspective suggested by Eq. (1) makes clear that transport coefficients in molecular liquids must be affected by a number of collective modes (number density, polarization density, etc.) and by different types of interaction potentials between the solute and the medium [van der Walls (vdW), electrostatic, surface hydrogen bonds, etc.]. On the other hand, the Stokes-Einstein phenomenology for diffusion and mobility in liquids, exploiting the paradigm of random molecular collisions, predicts a very generic view of the problem relating it solely to solute's geometry and solvent's shear viscosity $\eta$. Changes of coordinates of the tagged particle, e.g., its position and orientation, dissipate energy as prescribed by hydrodynamic equations and are all related to $\eta$.

This powerful paradigm leads to a number of relations between translation and rotation transport coefficients known as Stokes-Einstein-Debye (SED) relations [10,11]. When hydrodynamic equations are used to calculate the rotational relaxation time $\tau_{r}$ and the translational diffusion coefficient $D_{t}$ for a spherical solute with the radius $a$, the following relations follow [8,12]: $D_{t}=(6 \pi \beta \eta a)^{-1}$ and $\tau_{r}=4 \pi \beta \eta a^{3}$. From these equations, one can form the SED product

$$
D_{t} \tau_{r}=\frac{2}{3} a^{2} .
$$

The importance of this result is that it links single-particle rotations and translations trough the common dissipation mechanism: measuring one property is sufficient for knowing the other. 

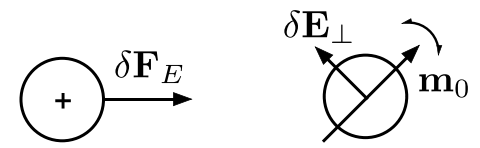

$$
\begin{array}{ll}
\delta \mathbf{F}_{E}=q_{0} \delta \mathbf{E}_{s} & \delta \mathbf{F}_{E}=0 \\
\delta \mathbf{T}_{E}=0 & \delta \mathbf{T}_{E}=\mathbf{m}_{0} \times \delta \mathbf{E}_{s}
\end{array}
$$

FIG. 1. Effect of electrostatic solute-solvent interactions on a spherical ion (charge $q_{0}$ ) and a spherical dipole (dipole moment $\mathbf{m}_{0}$ ). There is a fluctuating electrostatic force $\delta \mathbf{F}_{E}$ acting on the ion, but there is no electrostatic torque. In contrast, the force acting on the dipole is zero, but there is a nonzero electrostatic torque affecting the rotational dynamics if the solvent field has a projection $\delta \mathbf{E}_{\perp}$ perpendicular to the dipole $\mathbf{m}_{0}$.

Early warnings that this picture might be not entirely correct came from the classical work on dielectric friction [13-17]. The main message of those theories is that transport of a solute carrying an electric multipole is affected by its electrostatic interactions with the medium dipoles. Electrostatics is long-ranged and corresponding fluctuations involve many solvent molecules. Fundamentally, the notion of dielectric friction is a conceptual departure from the picture of individual molecular collisions. In contrast, it considers correlated subensembles of medium dipoles responding to the perturbation introduced by the solute with their collective relaxation time. The response of a polar medium to the solute movement is delayed, leading to dielectric loss. Dielectric friction is a result of this dissipation of energy in the material due to coupled collective movements of its dipoles.

The fluctuation-dissipation arguments [18] suggest that dissipation of energy due to dielectric loss is equivalent to fluctuations in the medium: the modes that produce most dissipation are those that fluctuate the most. One can, therefore, view the problem of dielectric friction in the light of fluctuating electrostatic forces acting from the solvent on the solute multipole. One immediately realizes that the results of such interactions will be entirely different for a spherical ion and for a spherical dipole.

Figure 1 illustrates the distinction between two configurations: (i) charge $q_{0}$ placed at the center of a spherical solute and (ii) the solute charge replaced with the point dipole $\mathbf{m}_{0}$. The fluctuation of the electrostatic force $\delta \mathbf{F}_{E}=q_{0} \delta \mathbf{E}_{s}$ produced by the fluctuating electric field of the solvent $\delta \mathbf{E}_{s}$ will contribute to the translational friction $\zeta_{t}=\left(\beta D_{t}\right)^{-1}$ a term proportional to $q_{0}^{2}$ [Eq. (1)] [19]. In contrast, rotational dynamics are affected by random torques $\mathbf{T}(t)$ acting from the medium on the solute [12]. If the charge is placed at the center of mass of the solute, then there will be no torque from electrostatic forces, $\mathbf{T}_{E}=0$ (Fig. 1). One, therefore, anticipates that electrostatics will affect $D_{t}$ but will not affect $\tau_{r}$. This disparity should lead to the SED violation.

Just the opposite situation applies to a point dipole. If the electric field is uniform within the solute, then there is no electrostatic force, $\mathbf{F}_{E}=0$, but there is an electrostatic torque,

$$
\mathbf{T}_{E}=\mathbf{m}_{0} \times \mathbf{E}_{s}
$$

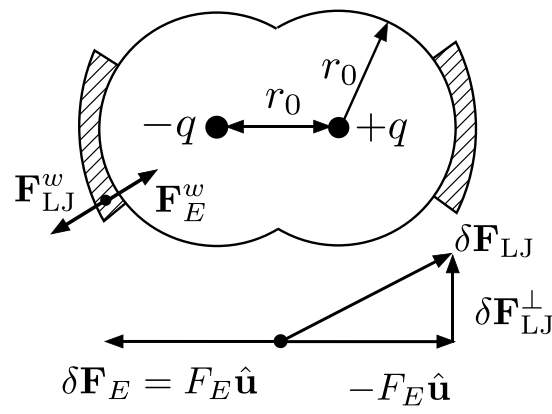

FIG. 2. Diagram of the dumbbell solute with charges $\pm q$ at the centers of two fused spheres. The shaded areas show arrested solvation layers at the surface of the solute, which rotate together with the solute. Equilibrium for the individual solvent molecules in this surface layer, $\mathbf{F}_{\mathrm{LJ}}^{w}=-\mathbf{F}_{E}^{w}$, projects into the condition $F_{\mathrm{LJ}}^{\|}=-F_{E}$ for the component of the vdW (LJ) force parallel to the unit vector $\hat{\mathbf{u}}$ specifying the solute orientation. Fluctuations of the vdW force in the perpendicular direction $\delta \mathbf{F}_{\mathrm{LJ}}^{\perp}$ contribute an additional component to the corresponding variance (see text for more detail).

The component of the solvent field perpendicular to the dipole $\delta \mathbf{E}_{\perp}$ (Onsager's directing field [20]) will rotate the dipole thus affecting the rotational dynamics (Fig. 1). Further, fluctuations $\delta \mathbf{T}_{E}$ will add friction to rotational relaxation [14] and alter $\tau_{r}$ without affecting $D_{t}$. One expects deviations from the hydrodynamic equations for $\tau_{r}$ while keeping $D_{t}$ consistent with hydrodynamics. Once again, a deviation from the SED relation should follow.

How these general arguments will actually translate to changes of transport coefficients of a probe molecule placed in a polar liquid is not easy to predict. This is because different interactions, mostly $\mathrm{vdW}$ and electrostatic interactions, compete in the overall time correlation functions constructed with forces and torques. Classical theories of dielectric friction assume that the effects of short-range vdW interactions and long-range electrostatics are separable, leading to additive components in the corresponding translational and rotational friction coefficients $[13,17]$. This assumption was shown to be incorrect for both the ion mobility [19,21-24] and rotational dynamics [25,26]. In this regard, it becomes important to study model situations in which electrostatic and vdW forces and torques can be well characterized.

We report here molecular dynamics (MD) simulations of a model solute with two opposite charges $+q$ and $-q$ placed symmetrically around the solute center of mass. The solute is composed of two fused Lennard-Jones (LJ) spheres with the radii $2.2 \AA$, which can be viewed as a model of an iodine molecule [26] (Fig. 2). The simulations done in roomtemperature simple point charge (SPC/E) force-field water aim at producing the dependence of rotational and translational dynamics of the solute on its dipole moment, which is altered by maintaining the distance between the charges while changing the partial charge $q$ in small steps. This setup has allowed us to test the basic predictions of classical theories of dielectric friction and contrast them with perturbation theories for the statistics of the electric field produced by the solvent inside the solute. 
The Nee-Zwanzig theory [14] predicts a linear scaling of $\tau_{r}$ with the squared dipole moment of the solute [Eq. (4)], which is approximately confirmed by our simulations. However, the SED product $D_{t} \tau_{r}$ never follows the prediction of Eq. (2) and instead is a linear function of $m_{0}^{2}$. The interaction of the solute dipole with the surrounding water molecules makes the translational diffusion coefficient in the body frame of the solute anisotropic. This anisotropy couples translations to rotations and leads to non-Gaussian translational dynamics on the timescale of rotational relaxation.

The violation of the SED relation and the non-Gaussian translational dynamics are often viewed as signature properties of low-temperature and supercooled liquids [27-30]. In contrast, our results for the transport coefficients are obtained in room-temperature water and show that this phenomenology arises from the electrostatic coupling of a tagged molecule with the medium. By extending these results to low temperatures, we suggest that explaining SED violation in supercooled water [31,32] requires development of physical models specifically including electrostatics in terms of dielectric friction.

\section{ROTATIONAL DYNAMICS}

Stochastic fluctuations of the torque initiated by fluctuations of the electric field [Eq. (3)] lead to the energy dissipation of the rotating dipole to the medium. This is the rotational dielectric friction, which slows the dipole rotation down. Fluctuation relations repeating the steps of Nee and Zwanzig [14] derivation are given in detail in Appendix C. They lead to the following equation for the integral rotational relaxation time

$$
\tau_{r}=\tau_{r}^{0}+\frac{\left(\beta m_{0}\right)^{2}}{6}\left\langle\left(\delta \mathbf{E}_{s}\right)^{2}\right\rangle \tau_{E} .
$$

In this equation, $\tau_{r}^{0}$ is the hydrodynamic rotational relaxation time and $\tau_{E}$ is the relaxation time of the electric field fluctuations. The product of the field variance $\left\langle\left(\delta \mathbf{E}_{s}\right)^{2}\right\rangle$ and $\tau_{E}$ is given by the time integral of the time correlation function of the field,

$$
\tau_{E}\left\langle\left(\delta \mathbf{E}_{s}\right)^{2}\right\rangle=\int_{0}^{\infty} d t C_{E}(t),
$$

where

$$
C_{E}(t)=\left\langle\delta \mathbf{E}_{s}(t) \cdot \delta \mathbf{E}_{s}(0)\right\rangle .
$$

Finally, the rotational relaxation time $\tau_{r}$ in Eq. (4) is calculated as the integral time based on the rotational time correlation function $C_{r}(t)$,

$$
\tau_{r}=\int_{0}^{\infty} d t C_{r}(t) .
$$

Here $C_{r}(t)$ is determined based on the dynamics of the unit vector $\hat{\mathbf{u}}(t)=\mathbf{m}_{0}(t) / m_{0}$ identified with the solute dipole

$$
C_{r}(t)=\langle\hat{\mathbf{u}}(t) \cdot \hat{\mathbf{u}}(0)\rangle .
$$

Equation (4) predicts slowing of the rotational dynamics, with the rotational relaxation time increasing linearly with the squared solute dipole $m_{0}^{2}$. This scaling is derived from the solvent linear response, when neither the variance of the electric

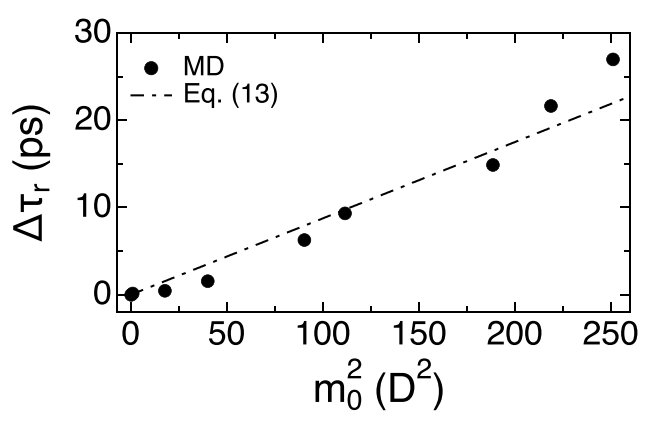

FIG. 3. Rotational relaxation time of the solute vs $m_{0}^{2}$. The dashed line shows the dielectric result from Eq. (13) calculated with $a=3 r_{0} / 16^{1 / 3}$.

field nor its relaxation time are affected by the presence of the solute. The rotational times of solutes with varying $m_{0}$ calculated from MD approximately follow the predicted scaling also reported in the past [26,33,34] (Fig. 3). The increment $\Delta \tau_{r}=\tau_{r}-\tau_{r}^{0}$ is shown in Fig. 3 , where the rotational time of the smallest dipole in our simulations, corresponding to $q=0.05$, is taken as the relaxation time $\tau_{r}^{0}$ unperturbed by electrostatics [Table I lists $\tau_{r}\left(m_{0}\right)$ ].

The MD protocol used in this study is explained in more detail in Appendix A. Important for the discussion is that SPC/E water at $T=300 \mathrm{~K}$ was used to solvate a dumbbell solute with the LJ radius $r_{0}=2.2 \AA$ close to the size of the iodide ion (Fig. 2). The solute interacts with water by two $\mathrm{LJ}$ potentials centered at the centers of two fused LJ spheres. The charges $+q$ and $-q$ are placed symmetrically around the particle's center of mass at the centers of two spheres separated by the distance $r_{0} ; q$ is listed here in elementary units of charge $e$. This simulation setup is close to that utilized by Kumar and Maroncelli [26].

Assuming linear response of the solvent allows us to connect fluctuations of the electric field to the susceptibility, i.e., the average field produced by the dipole in response to a small probe dipole. This average field, $R=\left\langle E_{s}\right\rangle=2 \chi_{R} m_{0}$, scaling linearly with the solute dipole, is known as the Onsager reaction field [20]. The linear susceptibility can be calculated in various formalisms including dielectric theories. The latter approach connects $\chi_{R}$ to the radius of the solute $a$ and the dielectric constant of the medium (solvent) $\epsilon_{s}$

$$
\chi_{R}=\frac{1}{a^{3}} \frac{\epsilon_{s}-\epsilon_{\infty}}{2 \epsilon_{s}+\epsilon_{\infty}},
$$

where the infinite-frequency dielectric constant $\epsilon_{\infty}$ is included to account for the solvent polarizability $\left(\epsilon_{\infty}=1\right.$ for SPC/E water used in simulations).

The reaction-field susceptibility $\chi_{R}$ can be alternatively expressed in terms of the variance of the electric field [19]

$$
\chi_{R}=(\beta / 6)\left\langle\left(\delta \mathbf{E}_{s}\right)^{2}\right\rangle .
$$

The rotational relaxation time becomes

$$
\tau_{r}=\tau_{r}^{0}+\beta m_{0}^{2} \chi_{R} \tau_{E} .
$$

The dynamic component of this equation, the relaxation time $\tau_{E}$ of the medium electric field inside the solute, also follows from dielectric theories [8,35] and is given in terms of the 
Debye relaxation time of the solvent $\tau_{D}$ (specified by the peak of the dielectric loss spectrum)

$$
\tau_{E}^{c}=\frac{3 \epsilon_{\infty}}{2 \epsilon_{s}+\epsilon_{\infty}} \tau_{D},
$$

where the superscript "c" marks the continuum estimate. The rotational relaxation time in the dielectric continuum estimate thus becomes [14,36,37]

$$
\tau_{r}^{c}=\tau_{r}^{0}+\tau_{D} \frac{3 \beta \epsilon_{\infty} m_{0}^{2}}{a^{3}} \frac{\epsilon_{s}-\epsilon_{\infty}}{\left(2 \epsilon_{s}+\epsilon_{\infty}\right)^{2}} .
$$

The dashed line in Fig. 3 shows the result of Eq. (13) with the dielectric cavity radius $a=3 r_{0} / 16^{1 / 3}$ made equal to the effective radius representing the volume of the fused diatomic. As we show below, a surprisingly good performance of the dielectric formula in reproducing the MD results in fact hides serious violations of the fundamental assumptions of the theory.

From the experimental standpoint, approximations inherent to dielectric theories can be avoided by linking the linear susceptibility $\chi_{R}$ to the Stokes shift and Stokes-shift dynamics provided by spectroscopy $[8,38]$. If the optical chromophore changes its dipole by $\Delta m_{0}$ in an optical transition, then the steady-state Stokes shift becomes $\Delta E^{\mathrm{St}}=2 \Delta m_{0}^{2} \chi_{R}$. This relation allowed van der Zwan and Hynes [39] to establish the connection between the excess of the relaxation time $\Delta \tau_{r}=$ $\tau_{r}-\tau_{r}^{0}$ and the Stokes shift

$$
\Delta \tau_{r}=\frac{m_{0}^{2}}{2 \Delta m_{0}^{2}} \beta \Delta E^{\mathrm{St}} \tau_{s},
$$

where $\tau_{s}=\tau_{E}$ is the integral Stokes-shift relaxation time defined, similarly to Eq. (5), by time integration of the corresponding time correlation function [37,40]. Equation (14) offers two significant advantages over Eq. (13): (i) no specific solvation model is required and (ii) the corrections of the dipole moment due to chromophore's polarizability [37,41] [often approximated as $(1 / 9)\left(\epsilon_{\infty}+2\right)^{2}$ ] cancel out in the ratio $m_{0} / \Delta m_{0}$.

A linear relation $\Delta \tau_{r} \propto \Delta E^{\mathrm{St}} \tau_{s}$ has been indeed observed experimentally $[37,41,42]$. However, the slope of this correlation was found to be grossly inconsistent with expectations based on the dipole moments $m_{0}$ and $\Delta m_{0}$ entering Eq. (14). This dramatic failure was related to the breakdown of the assumption of friction additivity inherent to both Eqs. (4) and (14) and to strong cross-correlations between vdW and electrostatic torques [26].

Equation (11) is derived under the assumption of linear response when neither the statistics nor the dynamics of the field fluctuations are affected by the solute. Both assumptions are violated with increasing solute dipole. The field variance is increased due to the density contraction of water around the solute caused by the electrostatic pull of the solute dipole (Fig. 19). The shell contraction can be partially accounted for by defining [19] an effective solute radius based on the solutesolvent radial distribution function (RDF) $g_{0 s}(r)$ (Fig. 19)

$$
a_{\text {eff }}^{-3}=3 \int_{0}^{\infty}\left(d r / r^{4}\right) g_{0 s}(r) .
$$

However, this radius contraction (filled points in Fig. 4) does not fully account for a nonlinear increase of the field fluc-

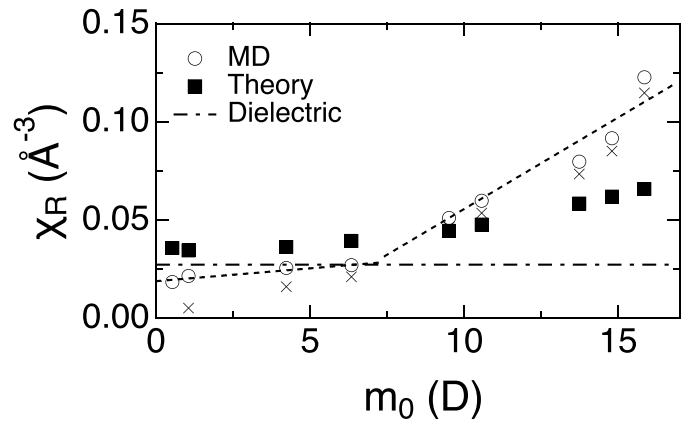

FIG. 4. Dipolar (reaction field) susceptibility calculated from MD [open points, Eq. (10)] and from an analytical model (filled points, Appendix B). The dash-dotted horizontal line refers to the dielectric estimate from Eq. (9) with $a=3 r_{0} / 16^{1 / 3}$. Crosses indicate $\chi_{R}$ calculated assuming that the field variance is produced by the solvent field projected on the direction of the solute dipole [Eq. (17)]. The dashed lines are linear fits through the simulation points.

tuations at higher solute dipoles. The results of calculations are slightly higher than simulations (open points in Fig. 4) at small solute dipoles, but fall below the simulation results at higher dipole values. However, an exact fit is not expected here since the calculations are done by adopting the field of a point dipole placed at the center of a spherical solute, while the simulated solute carries a nonpoint dipole with the field of the quadrupole moment affecting the result. The dielectric formula for $\chi_{R}$ [Eq. (9)] obviously does not account for all these complications, but provides a fair numerical estimate at small solute dipoles when linear response holds (dash-dotted line in Fig. 4).

The reaction-field susceptibility $\chi_{R}$ was calculated from Eq. (10) with the electric field $\mathbf{E}_{s}$ taken at the center of mass of the solute. This is in contrast to the calculation of electric forces discussed below, which are calculated from electric fields at the partial charges $q_{i}= \pm q$. The distribution of the electric field $p\left(\mathbf{E}_{s}\right)$ turns out to be strongly non-Gaussian when calculated in the laboratory frame (Fig. 5 shows the mean distribution $\left.\bar{p}=\frac{1}{3}\left[p\left(E_{s x}\right)+p\left(E_{s y}\right)+p\left(E_{s z}\right)\right]\right)$. This is the result of solute rotations, which transform the Gaussian distribution of the field in the body frame of the molecule

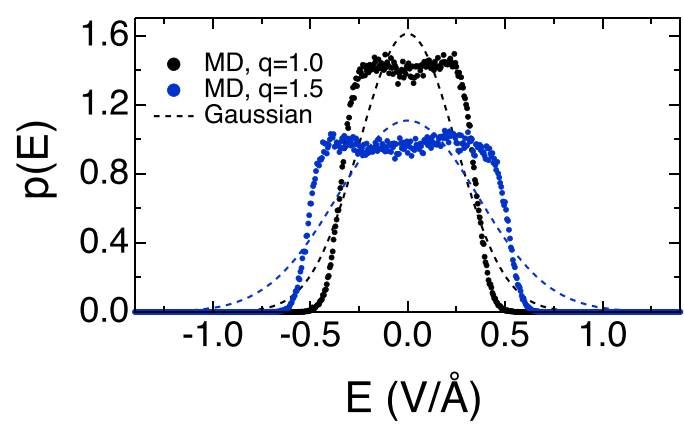

FIG. 5. Distribution of the Cartesian projections of the electric field created by SPC/E water at the geometrical center of the fused dumbbell solute. The simulation results (points) are compared to best fits to the Gaussian distribution (dashed lines). Calculations are done at $q=1.0$ and $1.5, T=300 \mathrm{~K}$. 


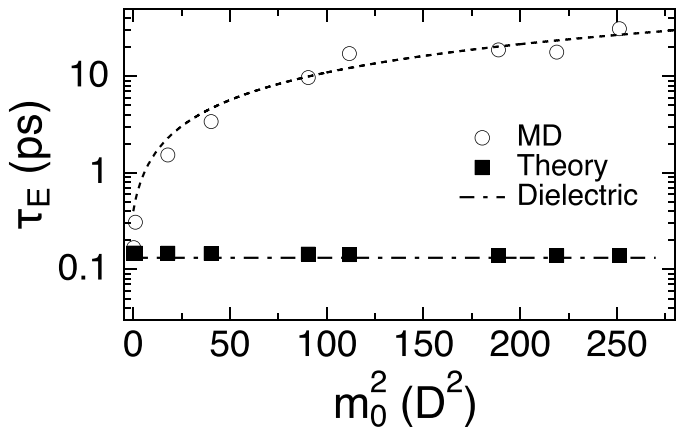

FIG. 6. Relaxation time $\tau_{E}$ calculated from MD (open points) and from the microscopic solvation theory (filled points, Appendix B). The dash-dotted line refers to the dielectric formula in Eq. (12) $\left(\tau_{D}=6.3 \mathrm{ps}\right.$ for SPC/E water at $300 \mathrm{~K}$ [43]). The dashed line is a linear fit through the MD points (note the logarithmic scale): $\tau_{E}=0.230+0.106 \times m_{0}^{2}$ ps, $m_{0}$ is in D.

(Fig. 21) to a stepwise distribution in the laboratory frame. A similar phenomenology, with the field distribution of a similar shape, is found for the electric field acting on a tagged molecule in the bulk SPC/E water (see below).

The idea that the variance of the solvent field $\mathbf{E}_{s}$ predominantly reflects solute rotations can be tested since in this case one can write the fluctuation of the field as

$$
\delta \mathbf{E}_{s}=\hat{\mathbf{u}}\left(R+\delta E_{\|}\right),
$$

where $R=\left\langle E_{\|}\right\rangle$is the reaction field [20] obtained by averaging the projection of the field on the direction of the dipole $E_{\|}=\hat{\mathbf{u}} \cdot \mathbf{E}_{s}$. The variance of the field in this approximation becomes

$$
\left\langle\left(\delta \mathbf{E}_{s}\right)^{2}\right\rangle \simeq R^{2}+\left\langle\left(\delta E_{\|}\right)^{2}\right\rangle .
$$

The field variance calculated by Eq. (17) is shown by crosses in Fig. 4. This approximation works increasingly well with increasing solute dipole, but becomes highly inaccurate at the lowest dipoles considered here. The reason for this change is that the solvent fluctuates independently of the solute rotations when the dipole is small, but becomes increasingly arrested at the solute surface when the solute dipole is increased (this is seen as the appearance of a shoulder in the radial distribution function shown in Fig. 19). Eventually, the surface layer rotates with the solute and the fluctuations of the field recorded in the laboratory frame are mostly due to solute rotations. The arrested layer needs to be understood as an arrested interfacial structure, in contrast to a stagnant surface layer or iceberg structure proposed in the past [44]. The orientational structure does not fluctuate much due to the electrostatic pull from the solute. The water molecules can still exchange between the interface and the bulk [45], but arrive to the interface in orientations specified by the local energetic preferences.

Consistent with this physical picture, the nonlinear effect of the solute dipole on the relaxation time of the electric field $\tau_{E}$ is much more pronounced than its effect on the statistics of field fluctuations (cf. Fig. 4 to Fig. 6). Microscopic calculations based on the linear response approximation [19] (filled points in Fig. 6, Appendix B) are consistent with the dielectric estimate in Eq. (12) (dash-dotted line, $\tau_{D}=6.3 \mathrm{ps}$ for SPC/E water [43]). Both formalisms fail to account for the retardation of the collective dynamics of the electric field reported by simulations. The increase of the dipole moment from $\simeq 0.5 \mathrm{D}$ at $q=0.05$ to $\simeq 16 \mathrm{D}$ at $q=1.5$ leads to a pronounced retardation of the electric field dynamics with the retardation factor of $\simeq 183$ (Table I). An approximately linear scaling of $\tau_{E}$ with $m_{0}^{2}$ is consistent with a similar trend for $\Delta \tau_{r}$ in Fig. 3 and we indeed find $\Delta \tau_{r} \approx \tau_{E}$ for large solute dipoles (Fig. 22). We can therefore conclude that the arrested structure of the water layer at the solute surface leads to a longer rotational time of the water-dressed solute and a good match between the rotational and electric field relaxation times.

Strong retardation of the field dynamics induced by the solute dipole brings the standard Nee-Zwanzig equation for the rotational dielectric friction [Eq. (11)] in significant disagreement with simulations. Retardation of rotational dynamics by the solute-solvent electrostatics as predicted by Eqs. (4) and (11) far exceeds the simulation results reported in Fig. 3 (the predicted rotational time becomes $\sim 800$ times higher than the MD result for the largest dipole studied here). It is clear that both dielectric and microscopic linear solvation theories do not capture this nonlinear retardation and the agreement between simulations and the dielectric formula shown in Fig. 3 is a result of strong cancellations of errors.

Past reports of the violation of linear response relations for dielectric friction [19,21-24,26] attributed it to crosscorrelations between electrostatic and LJ forces/torques. Here we also find significant cross-correlations between the LJ and electrostatic torques producing a negative contribution to the overall variance (Fig. 7, Appendix G)

$$
\left\langle(\delta \mathbf{T})^{2}\right\rangle=\left\langle\left(\delta \mathbf{T}_{E}\right)^{2}\right\rangle+\left\langle\left(\delta \mathbf{T}_{\mathrm{LJ}}\right)^{2}\right\rangle+2\left\langle\delta \mathbf{T}_{E} \cdot \delta \mathbf{T}_{\mathrm{LJ}}\right\rangle .
$$

We find that the variance of the electrostatic torque is remarkably close in magnitude to the negative of the cross-correlation between electrostatic and LJ torques (Tables II and III)

$$
-\left\langle\delta \mathbf{T}_{E} \cdot \delta \mathbf{T}_{\mathrm{LJ}}\right\rangle \simeq\left\langle\left(\delta \mathbf{T}_{E}\right)^{2}\right\rangle .
$$

One, therefore, can determine the torque variance as the difference between the LJ and electrostatic components

$$
\left\langle(\delta \mathbf{T})^{2}\right\rangle \simeq\left\langle\left(\delta \mathbf{T}_{\mathrm{LJ}}\right)^{2}\right\rangle-\left\langle\left(\delta \mathbf{T}_{E}\right)^{2}\right\rangle .
$$

If solute rotations do not involve memory effects, then the rotational relaxation time can be determined by integrating the torque-torque time correlation [7] function [cf. to Eq. (1)]

$$
\tau_{r}=\left(\beta^{2} / 6\right) \int_{0}^{\infty} d t\langle\delta \mathbf{T}(t) \cdot \delta \mathbf{T}(0)\rangle .
$$

Retardation of rotational relaxation can, therefore, be attributed to both the changes in the variance of the torque and changes in the relaxation time of the torque-torque correlation function. They both increase approximately linearly with $m_{0}^{2}$ and neither of them can be viewed as the sole factor responsible for retardation. Further, the relaxation times for electrostatic and cross correlation functions turn out to be very close. Combining this result with Eq. (19), the overall rotational time becomes the difference between the $\mathrm{LJ}$ and electrostatic components

$$
\tau_{r} \simeq \tau_{r}^{\mathrm{LJ}}-\tau_{r}^{E}
$$

where each relaxation time is calculated by integrating the corresponding component correlation function. In contrast, 

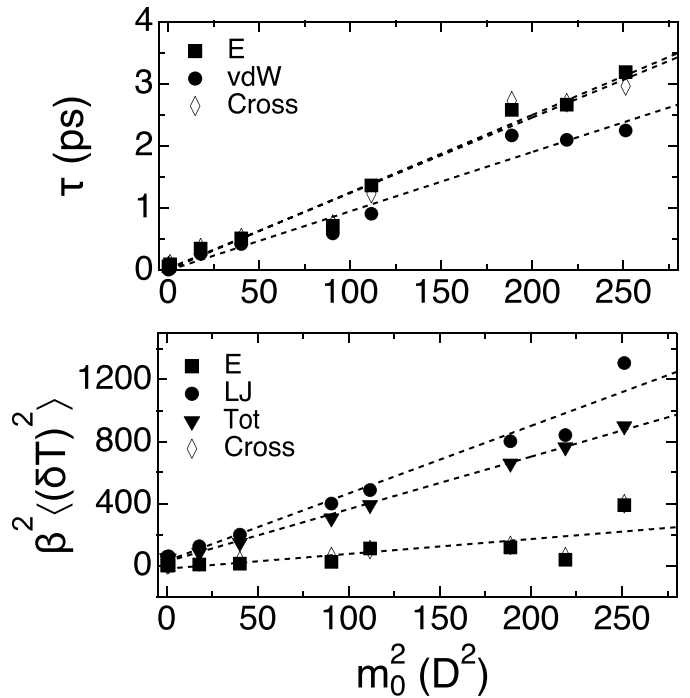

FIG. 7. Relaxation times (upper panel) and variances (lower panel) of the torque acting from water on the solute calculated from MD simulations (points). Electrostatic (E, filled squares), vdW (LJ, filled circles), and cross (Cross, open diamonds) components and total torque variance (Tot, filled triangle) in Eq. (18) are shown in the lower panel (Table III lists the numerical values). The average component relaxation times (obtained from normalized correlation functions) are shown in the upper panel, along with the correlation time of the cross-correlation component (cross, open diamonds). The dashed lines are linear fits through the points. The open diamonds nearly coincide with the filled squares on the scale of the plot; $T=300 \mathrm{~K}$.

the relaxation times shown in the upper panel of Fig. 7 are the average relaxation times of the corresponding normalized correlation functions.

In view of the surprising equality between the negative of the torque cross-correlation and the variance of the electrostatic torque, we additionally calculated the variance of the total force acting from water on the solute. The force is separated into the electric and LJ components, $\mathbf{F}=\mathbf{F}_{E}+\mathbf{F}_{\mathrm{LJ}}$ (Appendix E) and the force variance is separated into the electrostatic and LJ components similarly to Eq. (18) (Table II). We again find a nearly identical compensation between the electrostatic variance and the cross-correlation (Fig. 8)

$$
-\left\langle\delta \mathbf{F}_{E} \cdot \delta \mathbf{F}_{\mathrm{LJ}}\right\rangle \simeq\left\langle\left(\delta \mathbf{F}_{E}\right)^{2}\right\rangle
$$

This relation implies that, similarly to Eq. (20), the force variance can be approximated as the difference between the $\mathrm{LJ}$ and electrostatic self components

$$
\left\langle(\delta \mathbf{F})^{2}\right\rangle \simeq\left\langle\left(\delta \mathbf{F}_{\mathrm{LJ}}\right)^{2}\right\rangle-\left\langle\left(\delta \mathbf{F}_{E}\right)^{2}\right\rangle .
$$

The physical picture leading to this remarkable cancellation is discussed below.

\section{DIFFUSION ANISOTROPY}

The placement of a dipole at the solute makes the structure of water in the interface orientationally anisotropic. The solvent polarization induced by the dipole depends on the polar angle relative to the rotation axis. The solvent electrostatic

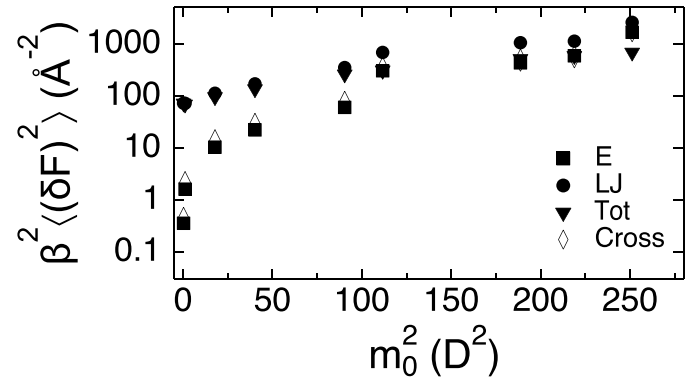

FIG. 8. Variance of the force acting from water on the solute from MD simulations (points). The variance is split into electrostatic (E, squares), vdW (LJ, circles), and cross-correlation (Cross, open diamonds) components (Table II lists of the numerical values). Filled triangles indicate the total variance; $T=300 \mathrm{~K}$.

response is also anisotropic. Given that translational diffusion is affected by both the interfacial structure and, through dielectric friction, by electrostatics, one can anticipate that the diffusion coefficient in the molecular frame of the solute gains anisotropy. The situation here is somewhat similar to the complex rotational/translational coupling of an ellipsoidal particle when anisotropic translational diffusion is caused by the particle shape [46-48]. We explore here the possibility of a similar phenomenology due to anisotropic interfacial polarization.

The anisotropic diffusion coefficient is calculated by rotating the vector of the particle velocity $\mathbf{v}(t)$ from the laboratory frame of the simulation box to the body frame fixed on the particle. The $z$ axis of the body frame is taken to coincide with the rotation axes specified by the unit vector $\hat{\mathbf{u}}(t)$ of the solute dipole. The body-frame velocity $\tilde{\mathbf{v}}(t)=\mathbf{R}(t) \cdot \mathbf{v}(t)$ is obtained by applying the rotation matrix $\mathbf{R}(t)$ aligning the $z$ axis with $\hat{\mathbf{u}}(t)$. The body-frame diffusion coefficients along the $z$ axis and perpendicular to it are calculated from time integrals of the velocity correlation functions

$\tilde{D}_{\|}=\int_{0}^{\infty} d t\left\langle\tilde{v}_{z}(t) \tilde{v}_{z}(0)\right\rangle, \quad D_{\perp}=\frac{1}{2} \int_{0}^{\infty} d t\left\langle\tilde{\mathbf{v}}_{\perp}(t) \cdot \tilde{\mathbf{v}}_{\perp}(0)\right\rangle$,

where $\tilde{\mathbf{v}}_{\perp}(t)=\tilde{\mathbf{v}}(t)-\hat{\mathbf{u}}(t) \tilde{v}_{z}(t)$.

The diffusion coefficient $\tilde{D}_{\|}$can be equivalently calculated in the laboratory frame by projecting the velocity on the direction of the dipole moment: $v_{u}(t)=\hat{\mathbf{u}}(t) \cdot \mathbf{v}(t)$. The diffusion coefficient becomes

$$
\tilde{D}_{\|}=\int_{0}^{\infty} d t\left\langle v_{u}(t) v_{u}(0)\right\rangle
$$

Given that linear velocities and orientations are statistically independent in the Gibbs ensemble, one can write

$$
\left\langle v_{u}(t) v_{u}(0)\right\rangle=\left\langle v_{\alpha}(t) v_{\beta}(0)\right\rangle\left\langle\hat{u}_{\alpha}(t) \hat{u}_{\beta}(0)\right\rangle,
$$

where summation over common Cartesian projections $\alpha, \beta$ is assumed. For isotropic rotational diffusion, one obtains [49]

$$
\left\langle\hat{u}_{\alpha}(t) \hat{u}_{\beta}(0)\right\rangle=\frac{1}{3} \delta_{\alpha \beta} C_{r}(t),
$$




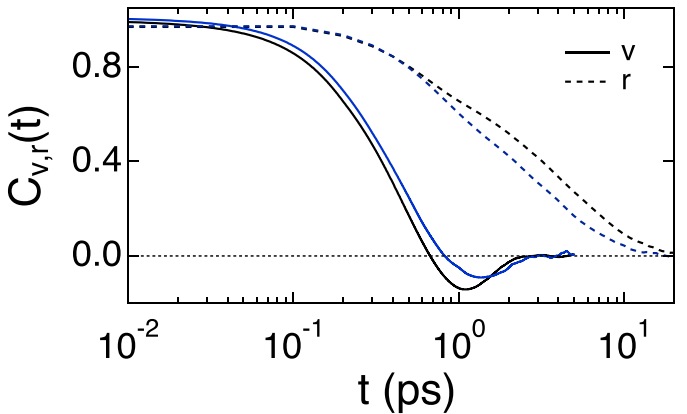

FIG. 9. $C_{v}(t)$ (solid lines) and $C_{r}(t)$ (dashed lines) for $q=0.4$, $m_{0}=4.2 \mathrm{D}$ (blue), and $q=0.6, m_{0}=6.3 \mathrm{D}$ (black). The dotted horizontal line marks the zero value. Both functions are normalized to their $t=0$ values.

where the rotational time correlation function is given by Eq. (8). The longitudinal diffusion coefficient finally becomes

$$
\tilde{D}_{\|}=\frac{1}{3} \int_{0}^{\infty} d t C_{v}(t) C_{r}(t) \simeq \frac{1}{3} \int_{0}^{\infty} d t C_{v}(t) e^{-t / \tau_{r}},
$$

where

$$
C_{v}(t)=\langle\mathbf{v}(t) \cdot \mathbf{v}(0)\rangle
$$

is the velocity time correlation function.

Equation (29) is a general result indicating that allowing the axis of symmetry to rotate modifies the diffusion coefficient along that axis. This is made clear in the second step in Eq. (29), where the rotational correlation function is replaced with a single-exponential decay with the rotational relaxation time $\tau_{r}$. Given the equality between physical body rotations and coordinate transformations [50], $\tau_{r}$ can be viewed as the timescale of laboratory frame rotations. Equation (29) shows that such random rotations of the laboratory frame affect the diffusion constant measured along a chosen axis of a rigid body. When $\tau_{r}$ much exceeds the relaxation time $\tau_{v}$ of the velocity correlation function, such frame rotations become insignificant and one restores the diffusion coefficient measured in the stationary laboratory frame.

The result of integration of two correlation functions in Eq. (29) depends on the relative amplitudes and time decays of the positive and negative portions of the velocity autocorrelation function. This is illustrated in Fig. 9, where we plot both $C_{v}(t)$ and $C_{r}(t)$. Because $\tau_{r}>\tau_{v}$, the decaying rotational correlation function mostly reduces the negative tail of $C_{v}(t)$ thus increasing the diffusion coefficient $\tilde{D}_{\|}$. This corresponds to $\tilde{D}_{\|}>\tilde{D}_{\perp}$ in Fig. 10. Rotations become slower and $\tau_{r}$ grows as the solute dipole is increased (Fig. 3). The negative portion of $C_{v}(t)$ contributes in this case more substantially to the integral, leading to closer values of the parallel and perpendicular diffusion coefficients in Fig. 10. One obtains nearly isotropic diffusion in the intermediate range of solute dipoles. Overall, the diffusion anisotropy $D_{\perp} / D_{\|}$scales linearly with $m_{0}^{2}$ (Fig. 10). We also find that $D_{\perp}$ stays nearly constant as $m_{0}$ is varied and the main effect of the dipole moment is on the parallel component $D_{\|}$(Fig. 26).

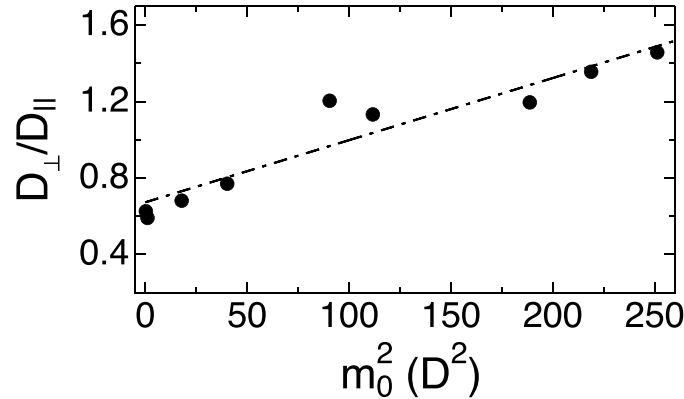

FIG. 10. Diffusion anisotropy in the body frame $\tilde{D}_{\perp} / \tilde{D}_{\|}$vs $m_{0}^{2}$ from MD simulations (points). The dash-dotted line is a linear fit.

\section{NON-GAUSSIAN TRANSLATIONAL DYNAMICS}

Anisotropic diffusion coefficient in the laboratory frame is responsible for rotation-translation coupling. This is already seen from Eq. (29) and the dependence of the diffusion anisotropy on the solute dipole in Fig. 10. Here, we present additional arguments for rotation-translation coupling by showing that translational dynamics carry the non-Gaussian character [47].

The translational and rotational degrees of freedom are coupled through the rotational matrix transformation involving a nonlinear, trigonometric dependence on the rotational angles [46-48]. This nonlinear coordinate transformation is responsible for non-Gaussian statistics of molecular displacements characterized by the non-Gaussian parameter $[51,52]$

$$
\alpha_{2}(t)=\frac{3\left\langle[\Delta \mathbf{r}(t)]^{4}\right\rangle}{5\left\langle[\Delta \mathbf{r}(t)]^{2}\right\rangle^{2}}-1,
$$

where $\Delta \mathbf{r}(t)$ is the center-of-mass displacement of the solute and $\Delta \mathbf{r}(t)^{4}=\left(\Delta r_{\alpha}(t) \Delta r_{\alpha}(t)\right)^{2}$.

To model $\alpha_{2}(t)$, one assumes that the solute Cartesian coordinate $r_{\alpha}(t)$ is described by the Langevin equation [47]

$$
\partial_{t} r_{\alpha}(t)=\xi_{\alpha}(t)
$$

where the random force is defined by the correlation function, $\left\langle\xi_{\alpha}(t) \xi_{\beta}\left(t^{\prime}\right)\right\rangle=2 D_{\alpha \beta}(t) \delta\left(t-t^{\prime}\right)$, incorporating the anisotropic diffusion constant

$$
\mathbf{D}(t)=D_{\|} \hat{\mathbf{u}}(t) \hat{\mathbf{u}}(t)+D_{\perp}[\mathbf{I}-\hat{\mathbf{u}}(t) \hat{\mathbf{u}}(t)] .
$$

From the Langevin equation, one obtains

$$
\left\langle\Delta r_{\alpha}(t) \Delta r_{\beta}(t)\right\rangle=2 \delta_{\alpha \beta} D_{\perp} t+2 \Delta D \int_{0}^{t} d t^{\prime}\left\langle\hat{u}_{\alpha}\left(t^{\prime}\right) \hat{u}_{\beta}\left(t^{\prime}\right)\right\rangle,
$$

where $\Delta D=D_{\|}-D_{\perp}$. From this equation and Eq. (28), the squared displacement of the solute is determined by the spherically symmetric diffusion coefficient $D_{t}=\bar{D}=\left(D_{\|}+\right.$ $\left.2 D_{\perp}\right) / 3$,

$$
\left\langle\Delta r^{2}(t)\right\rangle=6 \bar{D} t
$$

The mean-squared particle displacement does not register the translation-rotation coupling and that is why a measure of non-Gaussian dynamics in terms of higher statistical moments 


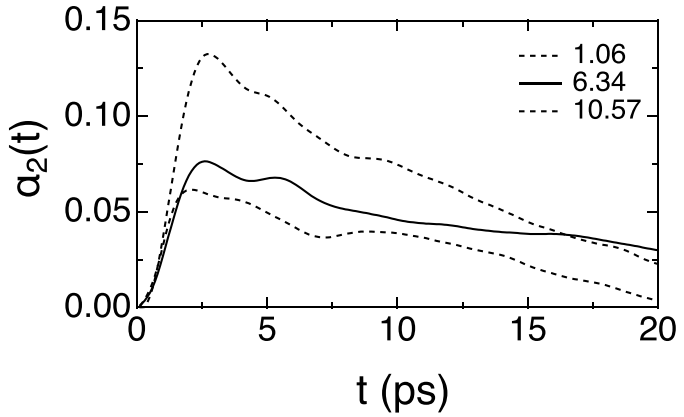

FIG. 11. Non-Gaussian translational dynamics parameter $\alpha_{2}(t)$ at $m_{0}$ values (D) listed in the plot. These values of the solute dipole correspond to partial charges $q=0.1,0.6$, and 1.0 placed at the fused solute dumbbell.

is required [53,54]. Assuming the mono-exponential decay of $C_{r}(t)$ with the relaxation time $\tau_{r}$, one obtains (Appendix $\mathrm{H}$ )

$$
\alpha_{2}(t)=\frac{2 \Delta D^{2}}{15 \bar{D}^{2}} f\left(3 t / \tau_{r}\right),
$$

where $f(x)=\left(x-1+e^{-x}\right) / x^{2}$. This equation applies to the long-time diffusional dynamics since no ballistic effects were included in Eqs. (32) and (35). It therefore does not reproduce the rising part of $\alpha_{2}(t)$ due to fast dynamics.

The calculated values of $\alpha_{2}(t)$ (Fig. 11) show slowly decaying tails, into tens of picoseconds, roughly consistent with the slow $\propto t^{-1}$ decay predicted by Eq. (36). The translational dynamics are non-Gaussian on the timescale of rotational relaxation (Fig. 3) roughly corresponding to the decay time seen in Fig. 11.

\section{STOKES-EINSTEIN-DEBYE RELATION}

A uniform electric field within a dipolar solute produces no force on the dipole (Fig. 1). Within this approximation, fluctuating electrostatic interactions do not impose dielectric friction on dipole translations, but significantly affect its rotations. This distinction is expected to alter the SED relation, which is based on pure hydrodynamic considerations and does not account for electrostatic effects.

The product $D_{t} \tau_{r}$ entering the SED equation is shown in Fig. 12. Equation (2) does not provide a quantitative estimate. An overestimated value of the SED product from Eq. (2) is

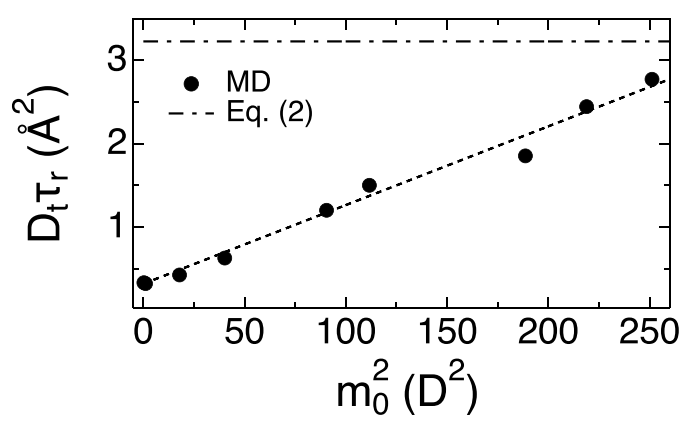

FIG. 12. The SED product [Eq. (2)] vs $m_{0}^{2}$. The dotted line indicates the SED result in the right-hand side of Eq. (2) with $a=r_{0}$. The dashed line is a linear fit through the simulation points.

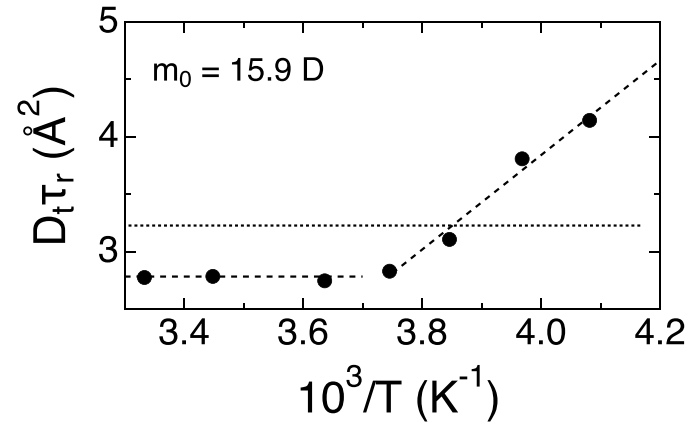

FIG. 13. The SED product for the dipolar fused dumbbell with $q=1.5$ vs the inverse temperature. The dots denote MD simulations and the dashed lines are linear fits of the corresponding portions of the data. The dotted line shows the result of Eq. (2).

also confirmed by the experimental results for water shown in Fig. 15 below. Instead of predicted constant value determined solely by the solute size, the SED product becomes an approximately linear function of $m_{0}^{2}$. This linear scaling mirrors the variation of $\tau_{r}$ shown in Fig. 3. SED violation is often related to transition from continuous diffusion to jumpdiffusion at low temperatures [55]. However, the concept of jump-diffusion does not apply to the bulky solute studied here and the SED violation is an electrostatic phenomenon.

Lowering temperature increases an effective strength of the solute-solvent dipolar coupling and can be mapped on an increase of the solute dipole. In fact, the violation of SED is most often recorded by lowering temperature [28]. Standard models of dielectric friction predict for the temperature variation

$$
\left[D_{t} \tau_{r}\right](T)=\frac{2}{3} a^{2}+\frac{m_{0}^{2} \chi_{R}}{6 \pi a} \frac{\tau_{E}(T)}{\eta(T)} .
$$

The second summand follows an Arrhenius temperature dependence arising from the field relaxation time and solvent viscosity, which often deviates from the Arrhenius law at low temperatures [56]. An important outcome here is that, depending on the relative activation energies of $\tau_{E}(T)$ and $\eta(T)$, the dielectric friction term will either increase or decrease with changing temperature. For the dipolar solute studied here, lowering temperature leads to a sharp rise of the SED product (Fig. 13). This temperature dependence is consistent with phenomenology typically reported for supercooled molecular glass-formers $[10,28,57]$.

Water is a good study case for SED violation by electrostatic interactions alone since almost the entire polarizability of water resides at the center of mass and no significant $\mathrm{vdW}$ torques are expected. This is confirmed by our direct calculations of the LJ and electrostatic torques acting on SPC/E water in the bulk (Table IV). Nevertheless, the direct application of Eq. (21) to calculate $\tau_{r}(T)$ gives overestimated results for SPC/E water, probably because water's rotations involve discontinuous jumps and cannot be reduced to rotational diffusion $[11,31,55,58,59]$.

We also find that the distribution of the electric field at a tagged molecule in bulk SPC/E water is highly non-Gaussian (Fig. 14). The origin of this peculiar distribution is the rotation of water in the laboratory frame, as is clarified for the 


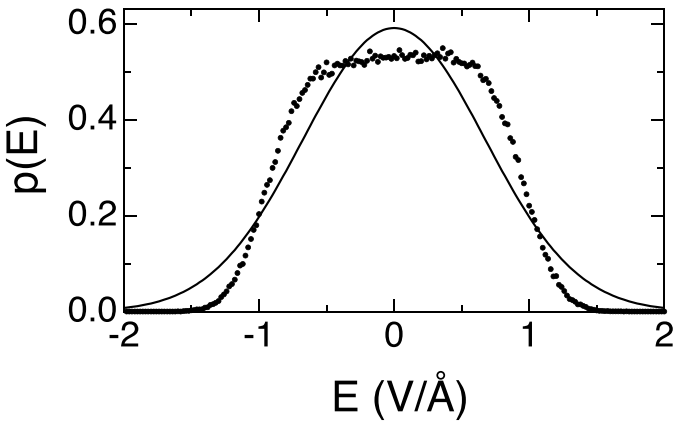

FIG. 14. Distribution of a Cartesian projection $E_{s \alpha}$ of the field at a tagged SPC/E water molecule (points). The simulation results are compared to the best fit to the Gaussian distribution (solid line); $T=320 \mathrm{~K}$.

dumbbell solute in Figs. 5 and 21. The distribution of the field is Gaussian in the body frame of the molecule, and it is shifted by the amount of the water reaction field [60] along the axis parallel to the molecular dipole. The electric field distribution is therefore Gaussian when projected on the $\mathrm{OH}$ bond. The corresponding field fluctuations strongly affect the vibrational band shape [61].

Rotations of water molecules in the laboratory frame spread the shifted Gaussian distributions along the dipolar axis into a stepwise functions with the range of electric-field magnitudes specified by the reaction field. Since transport coefficients and scattering of radiation are determined in the laboratory frame of reference, this observation implies that single-particle rotational dynamics of water affecting these observable properties should carry significant non-Gaussian features. We also find that the variance of the electric field at a tagged water molecule is an approximately linearly decaying function of temperature (Fig. 24), in direct violation of the expectations of the fluctuation-dissipation theorem [18] predicting $\propto T$ for the variance of a collective variable. Similar violations of the temperature law are found for the variance of the dipole moment of polar liquids [62].

Given the failure of the diffusion model for water's translations and rotations, one cannot expect that the present discussion can quantitatively resolve the problem of SED violation in low-temperature water. However, the strong effect of dipolar interactions in water on relaxation times and transport coefficients calls for inclusion of electrostatics in any considerations of water dynamics at low temperatures. Despite our observation that the direct use of Eq. (37) is not justified, supercooled water still shows the type of SED breakdown predicted by Eq. (37), which can be written as

$$
\left[D_{t} \tau_{r}\right](T)=A+B e^{\beta E_{a}} .
$$

Figure 15 shows the SED product for supercooled water [31,32], which fits well to Eq. (38). This functionality comes in contrast to the power law $\left[D_{t} \tau_{r}\right](T) \propto T^{-\gamma}$ suggested to fit the simulation results in Ref. [10]. In addition, two-state models of water thermodynamics have been used to describe the dependence of the water viscosity on temperature [63]. This approach was recently extended to rationalize effects of temperature and pressure on rotational relaxation and self-

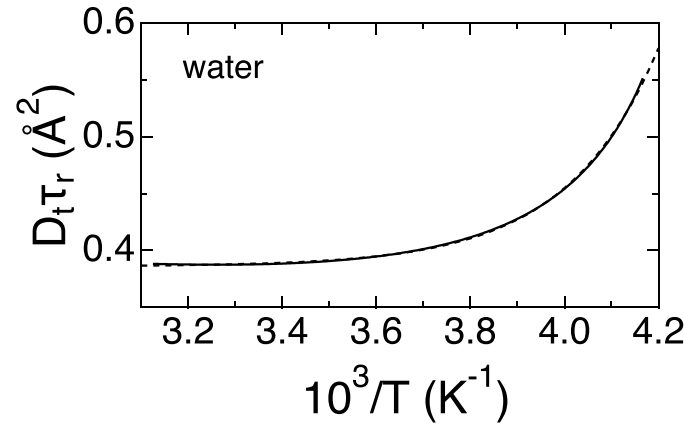

FIG. 15. The SED product for supercooled water [31,32]. The solid line is a numerical fit of experimental data from Ref. [32] and the solid line is the fit to the exponential function from Eq. (38) with $A=0.386 \AA^{2}, B=8.47 \times 10^{-11} \AA^{2}$, and $E_{a} / k_{B}=5129 \mathrm{~K}$.

diffusion [64,65]. However, the model is phenomenological and relies on a large number of fitting parameter.

There are a number of properties of force-field water models consistent with the phenomenology of dielectric friction affecting linear transport coefficients. The diffusion coefficient of force-field water was found to be anisotropic in the body frame [66,67], connecting to our calculations presented in Fig. 10. This result implies that phenomenology of non-Gaussian translational dynamics based on translationrotation coupling producing $\alpha_{2}(t) \neq 0$ (Fig. 11) applies to both high- and low-temperature water $[68,69]$. The strength of dipole-dipole interactions is determined by the dimensionless parameter $\left(m^{*}\right)^{2}=\beta m^{2} / \sigma_{s}^{3}$, where $m$ is the liquid dipole and $\sigma_{s}$ is the molecular diameter. Therefore, lowering temperature increases dielectric friction thus making $\alpha_{2}(t)$ higher in amplitude and shifting its decay to longer times $[10,68]$ because of slower rotations caused by increasing strength of dipoledipole interactions (Fig. 3).

\section{DISCUSSION}

Electrostatic solute-solvent interactions are viewed as a major reason for enhanced friction and slower translational $[19,21,22,24]$ and rotational $[25,26,33,34]$ diffusion of multipolar particles in polar media [14-17]. Dielectric friction is caused by fluctuations of the electric field produced by the solvent at the charge or dipole of the solute. The effect of electric field fluctuations on the rotational time of a dipolar solute is traditionally estimated through Eq. (4). A similar equation can be derived [19] for the translational diffusion coefficient $D_{t}$ of a spherical ion carrying the charge $q_{0}$ (in contrast to partial charges $\pm q$ making up the dipole, Fig. 1)

$$
D_{t}^{0} / D_{t}=1+\frac{\left(\beta q_{0}\right)^{2}}{3} \tau_{E} D_{t}^{0}\left\langle\left(\delta \mathbf{E}_{s}\right)^{2}\right\rangle,
$$

where $D_{t}^{0}$ is the Stokes-Einstein hydrodynamic diffusion coefficient. These two equations predict that combining rotational dynamics of a dipole with translational diffusion (conductivity) of an ion gives access to the same set of parameters describing the statistics and dynamics of the electric field in condensed materials. In particular, one can relate the diffusion coefficient of an ion to the increment $\Delta \tau_{r}$ of the rotational 
time of the dipole

$$
D_{t}^{-1}=\left(D_{t}^{0}\right)^{-1}+\frac{2 q_{0}^{2}}{m_{0}^{2}} \Delta \tau_{r} .
$$

The practicality of these predictions is severely diminished by two significant complications: (i) the competition and crosscorrelations between vdW (LJ) and electrostatic forces and torques $[19,26]$ and (ii) the nonlinear retardation of the field dynamics making $\tau_{E}$ a function of $q_{0}$ for an ion [19] and an approximately linear function of $m_{0}^{2}$ for the dipole (Fig. 6).

These two factors are so significant that they often invalidate Eqs. (4) and (39), as is the case with the present simulation setup. Both equations grossly overestimate the effect of electrostatics on either translational or rotational dynamics when accurate values of the field variance and relaxation time $\tau_{E}$ are applied. Consequently, the failure of Eq. (14) to describe experimental spectroscopic data $[37,41,42]$ is actually related to the failure of the standard linear theory of dielectric friction. From the experimental perspective, the effect of the solute dipole on the field dynamics shown in Fig. 6 can be translated to slower Stokes-shift relaxation for excited states of chromophores [70,71] usually carrying higher dipole moments. This observation also implies slower field dynamics in highly polar bulk liquids and inside solutes carrying large dipole moments, such as solvated proteins [72].

The range of dipole moments studied here exceeds what is typically found for spectroscopic dipolar probes of comparable sizes. To estimate how realistic these dipoles are and whether the strong retardation of the rotation dynamics and electric-field fluctuations can be achieved in real systems, one needs to consider the reduced solute dipole. The strength of dipole-water interaction is determined not by the dipole magnitude itself, but, instead, by the reduced dipole $\left(m_{0}^{*}\right)^{2}=\beta m_{0}^{2} / \sigma_{\text {eff }}^{3}$, where $\sigma_{\text {eff }}=2 a_{\text {eff }}$ is the effective diameter of the solute [Eq. (15), Figs. 19 and 20]. The reduced dipole $m_{0}^{*}$ reaches the value of $\simeq 5.4$ at the largest dipole moment explored in our simulations. Strong deviations between our calculations for rotational friction and standard theories start at $m_{0}^{*} \simeq 1$. For comparison, the reduced dipole of SPC/E water is $m^{*} \simeq 2.4$, where $\left(m^{*}\right)^{2}=\beta m^{2} / \sigma_{s}^{3}$ for a material with the molecular dipole $m$ and the molecular diameter $\sigma_{s}$ [5,74]. One therefore anticipates strong deviations from the standard linear theories of dielectric friction and dielectric relaxation for bulk water.

This expectation is supported by simulations of SPC/E water at different temperatures performed in this study. We find that Eq. (4) strongly overestimates the rotational time for water (Fig. 25). We also find that the continuum estimate $\tau_{E}=$ $\tau_{E}^{c}$ for the electric field relaxation [Eq. (12)] is much below $\tau_{E}$ reported by $\mathrm{MD}$ (Fig. 16). In contrast, $\tau_{E}(T)$ is slightly below the Debye relaxation time $\tau_{D}(T)$ for SPC/E water [73]. These results are consistent with the nonlinear retardation of the field dynamics shown in Fig. 6. The relaxation time deviates upward from $\tau_{E}^{c}$ with increasing dipole thus making $\tau_{D}(T)$ a better estimate of $\tau_{E}(T)$. This outcome might be of general importance for polar liquids, especially at low temperatures, when $m^{*}$ becomes larger in magnitude.

Another class of important solutes is colloidal particles, such as proteins, which are stabilized in solution by high

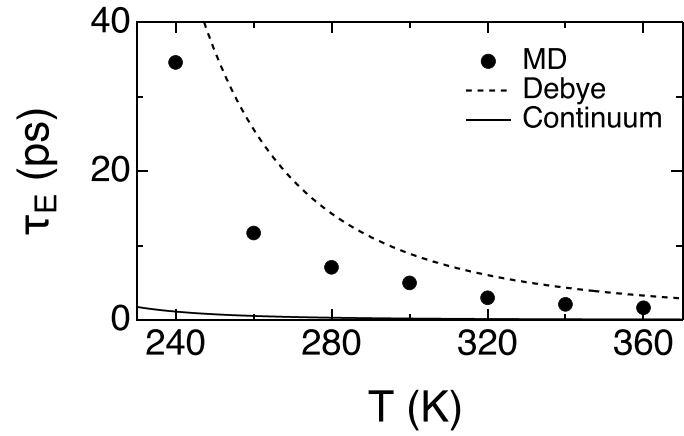

FIG. 16. $\tau_{E}$ from MD (points, MD) compared to $\tau_{D}$ of SPC/E water [73] (Debye) and to $\tau_{E}^{c}$ from Eq. (12) (Continuum).

density of surface charges. For proteins carrying significant dipole moment due to ionized surface residues [75], the reduced dipole $m_{0}^{*} \simeq 5$ is often found. This is close to the upper end of our scale and one should anticipate a dramatic failure of standard theories of dielectric friction when applied to protein's rotational dynamics [76]. A strong, about three to four orders of magnitude [72], retardation of the dynamics of electric field produced by water inside the protein parallels the field retardation found for the dipolar solutes in this study (Fig. 6).

What has been missing from previous studies of diffusion and mobility is the appreciation that electrostatic interactions bring to linear transport coefficients a number of features typically associated with the dynamics of low-temperature or supercooled liquids. We showed here that the diffusion coefficient of a dipolar solute is anisotropic in the body frame. Changing the solute dipole alters the diffusion anisotropy and brings it, for a large dipole, to the magnitudes comparable to anisotropy of a thin long $\operatorname{rod}\left(1 \leqslant D_{\|} / D_{\perp} \leqslant 2[49]\right)$. Because the coordinate transformation from the body frame to the laboratory frame is achieved by rotational matrices involving nonlinear trigonometric functionalities in terms of rotational angles, diffusion anisotropy is translated to nonGaussian translational dynamics on the timescale comparable to the rotational time [47]. In accord with the logic established in studies of dynamics of glass formers [53], one needs a four-point correlation function $\left[\alpha_{2}(t)\right.$ here] to interrogate the non-Gaussian dynamics. Further, distinct effects of electrostatics on translational and rotational dynamics (Fig. 1) lead to the violation of the SED relation linking the one-particle translational and rotational transport coefficients [Eq. (2); note that we avoid linking either of them to the collective property of shear viscosity].

The SED violation is often attributed to heterogeneous dynamics $[28,30,53,77]$, i.e., the temporal coexistence of domains with fast and slow dynamics in a homogeneous medium. In this framework, dynamic heterogeneity becomes a dynamic signature of nonergodicity in respect to experiments recording structural $\alpha$ relaxation. The appearance of distributed $\alpha$-relaxation times, leading to dispersed dynamics and non-Arrhenius kinetics [30,57,77], is the consequence of existence of a slower dynamic process (rate exchange) restoring ergodicity on a timescale much longer than $\alpha$ relaxation. That model explains SED violation by the fact that 
translational diffusion is dominated by fast states, while slow states mostly contribute to rotational diffusion [77] (this view was disproved in simulations of SPC/E water [10]). There are obviously no such distributed states in our relatively short simulations containing a single solute in the simulation cell, but we nevertheless find the same phenomenology of the SED product increasing either with solute polarity (Fig. 12) or with lowering temperature (Fig. 13).

In contrast to this "dynamical disorder" mechanism [78] for SED violation requiring introducing an unspecified slow rate exchange process, well-defined physical interactions are considered here to produce similar effects. Electrostatics affects translational diffusion of ions and rotation of dipoles, but does not affect rotations of ions and translations of dipoles (Fig. 1). As a result of this asymmetry, SED violation must occur when dielectric friction is significant. Our simulations indicate that this is true for strongly polar liquids at room temperature and must be true for essentially all dipolar liquids at low temperatures. This is because the strength of dipolar interactions $m^{*} \propto T^{-1 / 2}$ increases with lowering temperature and dielectric friction starts affecting linear transport coefficients at $m^{*}>1$. The SED relation assumes uncorrelated motion of single particles in a liquid. Electrostatics instead makes the particle motions strongly correlated and thus collective. The role of lowering temperature is to enhance these collective correlations.

Random torques responsible for rotational diffusion and random forces producing translational diffusion include vdW (LJ) and electrostatic components. They turn out to be remarkably correlated such that the negative of the cross-correlation of electrostatic and LJ force/torque is nearly exactly equal to the positive self-variance of the electrostatic component [Eqs. (19) and (23)]. This interesting phenomenon can be qualitatively explained by the diagram shown in Fig. 2.

Consider a thin layer of water at the solute surface. At large solute dipoles, the water layer is immobilized (shaded areas in Fig. 2) and, as we have shown above, rotates together with the solute (we still stress that it is the interfacial structure that is frozen, not the interfacial mobility of water molecules). For water molecules in these areas, equilibrium is established between the electrostatic pull and the LJ repulsion, $\mathbf{F}_{E}^{w}=-\mathbf{F}_{\mathrm{LJ}}^{w}$. Taken together, these individual equilibrium conditions imply $\delta \mathbf{F}_{E}=F_{E} \hat{\mathbf{u}}[$ Eq. (16)] and

$$
\delta \mathbf{F}_{\mathrm{LJ}}=-F_{E} \hat{\mathbf{u}}+\delta F_{\mathrm{LJ}}^{\perp} \hat{\mathbf{u}}_{\perp},
$$

where $\hat{\mathbf{u}}_{\perp}$ is the unit vector specifying the perpendicular projection of the vdW force. This latter relation immediately leads to the condition of equality between the cross-correlation and the negative variance of the electrostatic force [Eq. (23)]. Fluctuations of water density in the interface produce, in addition, fluctuations of the vdW force $\delta F_{\mathrm{LJ}}^{\perp}$ in the direction perpendicular to the solute dipole moment. The variance of the perpendicular vdW force contributes to the total variance making $\left\langle\left(\delta \mathbf{F}_{\mathrm{LJ}}\right)^{2}\right\rangle$ distinct from the variance of the electrostatic force $\left\langle\left(\delta \mathbf{F}_{E}\right)^{2}\right\rangle \simeq\left\langle F_{E}^{2}\right\rangle$ dominated by solute rotations. Density fluctuations thus enhance $\left\langle\left(\delta \mathbf{F}_{\mathrm{LJ}}\right)^{2}\right\rangle$ relative to $\left\langle\left(\delta \mathbf{F}_{E}\right)^{2}\right\rangle$. This phenomenology is illustrated in Fig. 17 where distributions of electrostatic forces parallel and perpendicular to the direction of the dipole unit vector $\hat{\mathbf{u}}$ are shown. The vertical dashed lines in the figure indicate the average

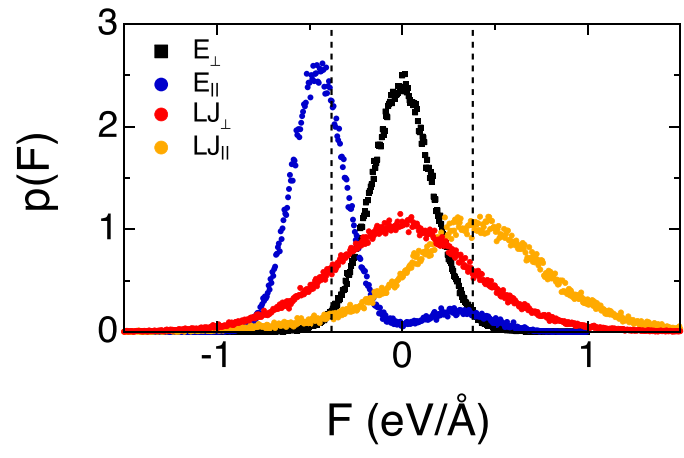

FIG. 17. Distribution of force projections on the direction of the solute dipole $\left(\mathrm{E}_{\|}\right.$and $\left.\mathrm{LJ}_{\|}\right)$and perpendicular to the solute dipole $\left(\mathrm{E}_{\perp}\right.$ and $\mathrm{LJ}_{\perp}$ ). The dashed vertical lines show the average parallel projections of the corresponding components. The results of simulations are shown at $q=1.3, m_{0}=13.7 \mathrm{D}$, and $T=300 \mathrm{~K}$.

parallel force projections, which are equal in magnitude and carry opposite signs to ensure the equilibrium condition.

The compensatory correlation between the forces was previously reported for a dipolar diatomic similar to the one studied here at a single dipole magnitude [26]. Similar remarkable equality between the variance of electric forces and the negative of the cross-correlation between $\mathrm{LJ}$ and electric forces was also found in our study of translational diffusion of solvated proteins [76]. Such a broad range of solutes potentially points to universality of this phenomenon, at least in application to diffusion of sufficiently large and highly polar solutes in water (it does not apply to water itself). Our results indicate that this phenomenology requires the formation of an arrested solvation layer at the solute surface.

\section{CONCLUSIONS}

Numerical simulations of dipolar dumbbells carried out here have shown a strong effect of the solute dipole on its rotational dynamics. Even though qualitatively predicted by classical theories of dielectric friction, the effect of electrostatics on linear transport coefficients is very different from these predictions. Two physical phenomena are at the center of this discrepancy: (i) nonlinear slowing down of the electric field dynamics of the polar medium and (ii) strong correlations between vdW and electrostatic solute-solvent interactions. The rotational dynamics of a dipolar solute in room-temperature water carries many qualitative signatures of linear transport coefficients in supercooled liquids: violation of the SED relation and non-Gaussian translational dynamics.

\section{ACKNOWLEDGMENT}

This research was supported by the National Science Foundation (Grant No. CHE-1800243) and through XSEDE resources (Grant No. TG-MCB080071).

\section{APPENDIX A: SIMULATION PROTOCOL}

The solute is a dumbbell made of two overlapping spheres with charges $+q$ and $-q$ at their centers. The radius of each sphere, $r_{0}=2.2 \AA$, defines the LJ diameter $\sigma_{0}=2 r_{0}=4.4 \AA$ 


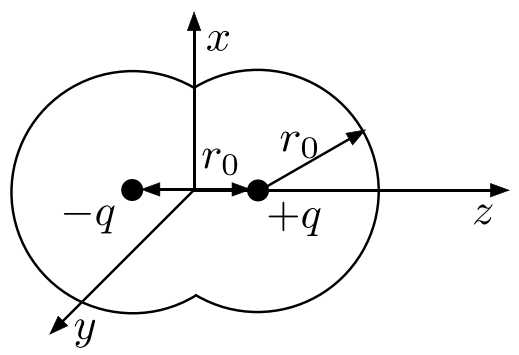

FIG. 18. Configurations of charges in the dumbbell solute. Also shown is the body frame associated with the solute.

for the repulsive core of the spheres. The $\mathrm{LJ}$ interaction potential between the solute and the oxygen atom of SPC/E water is given as a sum of two LJ site-site potentials

$$
U_{\mathrm{LJ}}(\mathbf{r})=4 \epsilon_{\mathrm{LJ}} \sum_{i}\left[\frac{\sigma_{\mathrm{LJ}}^{12}}{\left|\mathbf{r}-\mathbf{s}_{i}\right|^{12}}-\frac{\sigma_{\mathrm{LJ}}^{6}}{\left|\mathbf{r}-\mathbf{s}_{i}\right|^{6}}\right],
$$

where $\mathbf{s}_{i}, i=1,2$ are the vectors $s=\left|\mathbf{s}_{i}\right|=r_{0} / 2$ pointing from the geometric center of the solute (origin of the body frame, Fig. 18) to the centers of two spheres. The solutesolvent LJ diameter is $\sigma_{\mathrm{LJ}}=3.783 \AA$ and the solute-solvent $\mathrm{LJ}$ energy is $\epsilon_{\mathrm{LJ}}=0.12579 \mathrm{kcal} / \mathrm{mol}$. For SPC/E water, the LJ diameter for the oxygen-oxygen interaction is $\sigma_{\mathrm{LJ}}=3.166 \AA$ and the LJ energy is $\epsilon_{\mathrm{LJ}}=0.15535 \mathrm{kcal} / \mathrm{mol}$ [79]. The distance between the centers of spheres in the dumbbell is $2 s=$ $r_{0}$. Each sphere carries the mass of 126.9 a.u. thus making the total mass of the solute equal to 253.8 a.u.

The Large scale Atomic/Molecular Massively Parallel Simulator MD package was used for the production of trajectories. We have followed the standard protocol of MD integration with the integration time-step of $1 \mathrm{fs}$, NVT ensemble at $T=300 \mathrm{~K}$, and the SPC/E force field for water.

A cubic simulation box with length $L=35.2 \AA$ was used with periodic boundary conditions applied in all three directions. The solute was solvated with 1500 water molecules. The PPPM Ewald method was applied to compute electrostatic interactions and a $14 \AA$ cutoff was used for nonbonded interactions. All systems were equilibrated for $10 \mathrm{~ns}$, followed by production runs for $100 \mathrm{~ns}$ for smaller charges and $200 \mathrm{~ns}$ for $q \geqslant 1.0$. A number of dipole moments of the solute were examined by altering the opposite charges while keeping the distance between them constant (Table I); the charge is given in elementary charge units $e$.

We have additionally performed simulations of bulk SPC/E water at $T=240,260,280,300,320,340,360 \mathrm{~K}: 4700$ water molecules were placed in a cubic box with the side length of $52 \AA$ and simulated for $100 \mathrm{~ns}$ in the NVT ensemble following $10 \mathrm{~ns}$ equilibration in the NPT ensemble (at $1 \mathrm{~atm}$ pressure). The integration step was 1 fs and the saving time was 0.1 ps. The bond angle and the bond distance of water molecules are kept fixed using the SHAKE algorithm [80]. The PPPM Ewald method was used to compute electrostatic interactions and a $10 \AA$ cutoff was applied to LJ interactions.

\section{APPENDIX B: LINEAR RESPONSE THEORY}

The solute-oxygen RDFs $g_{0 s}(r)$ are shown in Fig. 19, where the distance $r$ is between the geometric center of the
TABLE I. Rotational relaxation time $\tau_{r}$, relaxation time of the electric field $\tau_{E}$, the translational diffusion coefficient $D_{t}\left(\AA^{2} / \mathrm{ps}\right)$ and the electric field variance $\left[(\mathrm{V} / \AA)^{2}\right]$ for solutes with changing partial charge $q$ and the corresponding dipole moment $m_{0} ; T=300 \mathrm{~K}$.

\begin{tabular}{lccccc}
\hline \hline$q(\mathrm{e})$ & $m_{0}(\mathrm{D})$ & $\tau_{r}(\mathrm{ps})$ & $\tau_{E}(\mathrm{ps})$ & $D_{t}$ & $\left\langle\left(\delta \mathbf{E}_{s}\right)^{2}\right\rangle$ \\
\hline 0.05 & 0.5 & 2.26 & 0.17 & 0.1484 & 0.041 \\
0.1 & 1.1 & 2.40 & 0.31 & 0.1352 & 0.048 \\
0.4 & 4.2 & 2.71 & 1.5 & 0.1577 & 0.057 \\
0.6 & 6.3 & 3.82 & 3.4 & 0.1649 & 0.060 \\
0.9 & 9.5 & 8.54 & 9.7 & 0.1409 & 0.114 \\
1.0 & 10.6 & 11.58 & 17.2 & 0.1299 & 0.133 \\
1.3 & 13.7 & 17.13 & 18.8 & 0.1083 & 0.178 \\
1.4 & 14.8 & 23.89 & 17.8 & 0.1024 & 0.205 \\
1.5 & 15.9 & 29.22 & 31.1 & 0.0949 & 0.274 \\
\hline \hline
\end{tabular}

solute and the oxygen atom. The RDFs indicate a continuous compression of the hydration shell as the dipole moment of the solute is increased. They also indicate anisotropy growing with increasing charge $q$, as is seen from a shoulder appearing in the first peak (blue and green lines in Fig. 19).

The effective solute radius is calculated from the RDF according to Eq. (15). The radii $a_{\text {eff }}$ are plotted in Fig. 20 as a function of the site charge $q$. At the smallest charge studied here, $a_{\text {eff }}$ is slightly below the solute-water LJ distance $\sigma_{\mathrm{LJ}}=$ $3.783 \AA$. The effective radius is always below the position of the first maximum of the RDF also shown in the figure.

The variance of field fluctuations was calculated from linear response theory as described in Ref. [19] and briefly reproduced here. The reaction-field static susceptibility $\chi_{R}$ follows from the linear-response equation [81]

$$
\chi_{R}=(\beta / 2) \int_{\Omega} d \mathbf{r} d \mathbf{r}^{\prime} E_{\mu}^{\alpha}(\mathbf{r})\left\langle\delta P^{\alpha}(\mathbf{r}) P^{\beta}\left(\mathbf{r}^{\prime}\right)\right\rangle E_{\mu}^{\beta}\left(\mathbf{r}^{\prime}\right) .
$$

The correlation of polarization fluctuations at points $\mathbf{r}$ and $\mathbf{r}^{\prime}$ in the liquid requires accounting for the presence of the solute, which imposes strong nonlinear effect on the Gaussian correlation functions through the requirement for the polarization field to vanish within the solute [82]. This problem has a closed-form solution for a spherical dipole [83] with the result

$$
\chi_{R}=\frac{3}{2 \epsilon_{s}+1}\left[\epsilon_{s} \chi_{R}^{L}+\chi_{R}^{T}\right]
$$

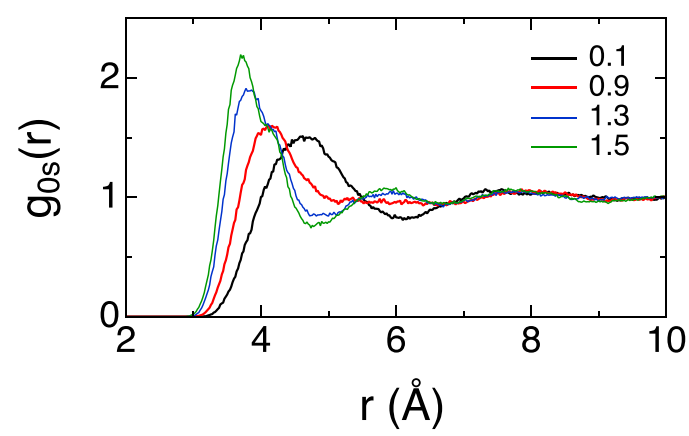

FIG. 19. RDFs between the dipolar solute and water's oxygen at different values of the partial charge $q$ listed in the plot. 


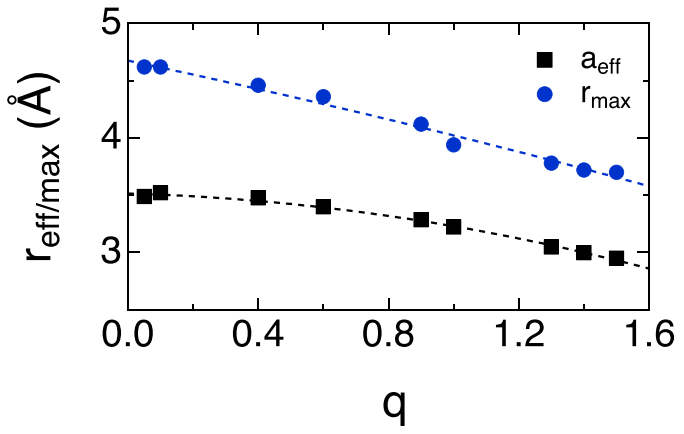

FIG. 20. RDF first maxima $\left(r_{\max }\right)$ and effective radii $\left(a_{\text {eff }}\right)$ calculated from Eq. (15).

Here, the longitudinal (L) and transverse (T) susceptibilities are given as reciprocal-space integrals of the squared field of the solute dipole with the corresponding longitudinal and transverse dipolar susceptibilities $\chi_{R}^{\gamma}(k)$ of bulk polar liquid

$$
\chi_{R}^{\gamma}=\frac{1}{2} \int \frac{d \mathbf{k}}{(2 \pi)^{3}} \chi^{\gamma}(k)\left|\tilde{E}_{\mu}^{\gamma}(\mathbf{k})\right|^{2},
$$

where $\gamma=\mathrm{L}, \mathrm{T}$.

The susceptibility $\chi_{R}$ corresponds to a unit dipole placed at the center of a spherical solute with the radius $a$. The reciprocal space electric field of the unit dipole is

$$
\tilde{\mathbf{E}}_{\mu}(\mathbf{k})=-\frac{4 \pi j_{1}(k a)}{k a}[3 \hat{\mathbf{k}}(\hat{\mathbf{k}} \cdot \hat{\mathbf{z}})-\hat{\mathbf{z}}],
$$

where the dipole is oriented along the $z$ axis (Fig. 18) and $j_{1}(x)$ is the first-order spherical Bessel function [84]. The longitudinal and transverse components of the field are obtained by taking the fields projections along the wave vector $\hat{\mathbf{k}}=\mathbf{k} / k$ and perpendicular to it: $\tilde{E}_{\mu}^{L}=\hat{\mathbf{k}} \cdot \tilde{\mathbf{E}}_{\mu}$ and $\tilde{\mathbf{E}}_{\mu}^{T}=\tilde{\mathbf{E}}_{\mu}-\hat{\mathbf{k}} \tilde{E}_{\mu}^{L}$. The calculations of $\chi_{R}$ in Eq. (B3) were done by using the response functions of bulk SPC/E water calculated from separate MD simulations [85]

$$
\chi^{\gamma}(k)=(3 y / 4 \pi) S^{\gamma}(k) .
$$

Here $y=(4 \pi / 9) \beta \rho m^{2}$ and $S^{\gamma}(k)$ are the longitudinal and transverse structure factors of the molecular dipoles in SPC/E water

$$
\begin{aligned}
S^{L}(k) & =\frac{3}{N} \sum_{j, k}\left\langle\left(\hat{\mathbf{u}}_{j} \cdot \hat{\mathbf{k}}\right)\left(\hat{\mathbf{k}} \cdot \hat{\mathbf{u}}_{k}\right) e^{i \mathbf{k} \cdot \mathbf{r}_{j k}}\right\rangle, \\
S^{T}(k) & =\frac{3}{2 N} \sum_{j, k}\left\langle\left[\hat{\mathbf{u}}_{j} \cdot \hat{\mathbf{u}}_{k}-\left(\hat{\mathbf{u}}_{j} \cdot \hat{\mathbf{k}}\right)\left(\hat{\mathbf{k}} \cdot \hat{\mathbf{u}}_{k}\right)\right] e^{i \mathbf{k} \cdot \mathbf{r}_{j k}}\right\rangle,
\end{aligned}
$$

where $\hat{\mathbf{u}}_{j}$ are unit vectors of $N$ dipoles located at $\mathbf{r}_{j}$ and $\mathbf{r}_{j k}=\mathbf{r}_{j}-\mathbf{r}_{k}$.

The relaxation time of electric field fluctuations is calculated as explained in detail in Ref. [19]. This formalism involves calculation of reciprocal space integrals involving the longitudinal and transverse projections of the field and the corresponding projections of the structure factors

$$
\tau_{E}=\tau_{E}^{c} \frac{2 \epsilon_{s} I^{L}+\epsilon_{\infty}^{3} I^{T}}{2 \epsilon_{s} J^{L}+\epsilon_{\infty}^{3} J^{T}},
$$

where $J^{\gamma}$ and $I^{\gamma}(\gamma=\mathrm{L}, \mathrm{T})$ are given by expressions

$$
\begin{gathered}
J^{\gamma}=\frac{6 a}{\pi} \int_{0}^{\infty} d k j_{1}^{2}(k a) S^{\gamma}(k), \\
I^{\gamma}=\frac{6 a}{\pi} \int_{0}^{\infty} d k j_{1}^{2}(k a) \frac{\left(S^{\gamma}(k)\right)^{2}}{S^{\gamma}(0)} \frac{1}{1+p k^{2}} .
\end{gathered}
$$

The parameter $p$ in Eq. (B9) quantifies the effect of coupled translational and rotational motions of molecular dipoles [8]. It is given as the product of the self-diffusion coefficient $D_{t}$ of water with the rotational time $\tau_{r}: p=\tau_{r} D_{t}$. For SPC/E water one finds [19] $p=1.5 \AA^{2}$. Finally, $\tau_{E}^{c}$ in Eq. (B7) is the dielectric rotational time in Eq. (12) $\left(\tau_{D}=6.3\right.$ ps is adopted for the Debye relaxation time of SPC/E water at $298 \mathrm{~K}$ [43]). Finally, the high frequency dielectric constant $\epsilon_{\infty}$ is put equal to unity in all calculations with nonpolarizable SPC/E water.

\section{APPENDIX C: ROTATIONAL TIME}

Here we follow the steps by Nee and Zwanzig [14] to derive the rotational time affected by electrostatics [Eq. (4)]. The electrostatic torque acting on the solute dipole is

$$
\mathbf{T}_{E}=\mathbf{m}_{0} \times \mathbf{E}_{s},
$$

where $\mathbf{E}_{s}$ is the electric field of the solvent. In order to produce a nonzero torque, the field has to be out of equilibrium. To calculate the retardation of the solute field, one looks for the frequency-dependent field produced by the probe dipole $\mathbf{m}_{0}(t)$ oscillating with the frequency $\omega$ and the amplitude $\mathbf{m}_{\omega}[5]$

$$
\tilde{\mathbf{R}}(\omega)=\tilde{\chi}_{R}(\omega) \cdot \mathbf{m}_{\omega} .
$$

The tildes here denote the Fourier-Laplace transforms and $\tilde{\chi}_{R}(\omega)$ is a second-rank tensor. Note that $\chi_{R}$ in Eq. (B2) is the isotropic static limit of the susceptibility $\tilde{\chi}_{R}(\omega): \chi_{R}=$ $(1 / 6) \operatorname{Tr}\left[\tilde{\chi}_{R}(0)\right]$.

The torque at time $t$ is given as the inverse time transform

$$
\begin{aligned}
\mathbf{T}_{E}(t) & =\int_{-\infty}^{\infty} \frac{d \omega}{(2 \pi)} \mathbf{m}_{0}(t) \times \tilde{\chi}_{R}(\omega) \cdot \mathbf{m}_{\omega} e^{-i \omega t} \\
& =\int_{-\infty}^{\infty} \frac{d \omega}{(2 \pi)} \tilde{\chi}_{R}(\omega) \cdot \int_{-\infty}^{\infty} d t^{\prime} e^{-i \omega\left(t-t^{\prime}\right)}\left(\mathbf{m}_{0}(t) \times \mathbf{m}_{0}\left(t^{\prime}\right)\right) .
\end{aligned}
$$

Instantaneous rotations of the dipole are described [86] by the instantaneous angular velocity $\boldsymbol{\Omega}(t)$

$$
\dot{\mathbf{m}}_{0}=\boldsymbol{\Omega} \times \mathbf{m}_{0} .
$$

The solution of this equation is

$$
\mathbf{m}_{0}(t)=\mathbf{m}_{0}\left(t^{\prime}\right)+\int_{t^{\prime}}^{t} d s \boldsymbol{\Omega}(s) \times \mathbf{m}_{0}(s)
$$

This equation is substituted to Eq. (C3) to obtain the linear response of the torque to the dipole rotation. Two approximations are applied in the Nee-Zwanzig theory [14]: (i) $\tilde{\chi}_{R}(\omega)$ is viewed as the isotropic tensor multiplied by a scalar function $\tilde{\chi}_{R}(\omega)$ and (ii) only the terms linear in $\Omega$ are included (linear response). The result is

$$
\mathbf{T}(t)=i m_{0}^{2} \int_{-\infty}^{\infty} \frac{d \omega}{(2 \pi)} \boldsymbol{\Omega}_{\perp}(\omega) \frac{1}{\omega}\left[\tilde{\chi}_{R}(\omega)-\tilde{\chi}_{R}(0)\right] e^{-i \omega t}
$$


where $\boldsymbol{\Omega}_{\perp}(t)$ is the projection of the instantaneous rotation frequency perpendicular to $\mathbf{m}_{0}$ and $\boldsymbol{\Omega}_{\perp}(\omega)$ is its time Fourier transform.

Equation (C6) has the form of linear friction

$$
\mathbf{T}(\omega)=-\zeta_{D}(\omega) \boldsymbol{\Omega}_{\perp}(\omega)
$$

with the dielectric friction coefficient

$$
\zeta_{D}(\omega)=\frac{m_{0}^{2}}{i \omega}\left[\tilde{\chi}_{R}(\omega)-\tilde{\chi}_{R}(0)\right] .
$$

The rotational relaxation time is a sum of the relaxation time $\tau_{r}^{0}$ due to vdW interactions and the dielectric friction component

$$
\tau_{r}=\tau_{r}^{0}+\left.\frac{\beta m_{0}^{2}}{2} \operatorname{Im} \frac{\tilde{\chi}_{R}(\omega)-\tilde{\chi}_{R}(0)}{\omega}\right|_{\omega \rightarrow 0} .
$$

The function $\tilde{\chi}_{R}(\omega)$ can be calculated from the time correlation function of the electric field according to the equation [5]

$$
\tilde{\chi}_{R}(\omega)=\frac{1}{3} \beta\left\langle\left(\delta \mathbf{E}_{s}\right)^{2}\right\rangle\left[1+i \omega \tilde{\Phi}_{E}(\omega)\right],
$$

where $\tilde{\Phi}_{E}(\omega)$ is the Fourier-Laplace transform of the normalized time correlation function $\Phi_{E}(t)=C_{E}(t) / C_{E}(0)$ with $C_{E}(t)$ given by Eq. (6). Adopting a multiexponential decay for the field-field correlation function

$$
\Phi_{E}(t)=\sum_{i} a_{i} e^{-t / \tau_{i}}, \quad \sum_{i} a_{i}=1,
$$

one arrives at Eq. (4) with

$$
\tau_{E}=\sum_{i} a_{i} \tau_{i}
$$

given as the integral relaxation time of the electric field (Table I).

\section{APPENDIX D: STATISTICS AND DYNAMICS OF THE SOLVENT FIELD}

It is typically assumed that the statistics of the electric field created by a dense polar solvent inside the solute is Gaussian. This is often adopted without testing, but we found here that one has to discriminate between distributions of the electric field in the laboratory and body frames of the solute.

The calculations of the field statistics were first done in the laboratory frame. The results shown in Fig. 5 are for the mean distribution

$$
\bar{p}=\frac{1}{3}\left(p\left(E_{s x}\right)+p\left(E_{s y}\right)+p\left(E_{s z}\right)\right)
$$

averaged over three individual distributions of field projections to reduce sampling errors. The field $\mathbf{E}_{s}$ is calculated at the geometrical center of the solute.

The distribution of the field in the solute body frame is produced by rotating $\mathbf{E}_{s}$ from the laboratory frame to the body frame with the $z$ axis along the solute dipole (Fig. 18) for each configuration along the simulation trajectory. The distributions become Gaussian in this reference frame. The Gaussian is shifted along the dipole axis to the value of the average reaction field [20,60] of water (Fig. 21). The non-Gaussian distribution in the laboratory frame shown in Fig. 5 is therefore a result of solute rotations, with the maxi-

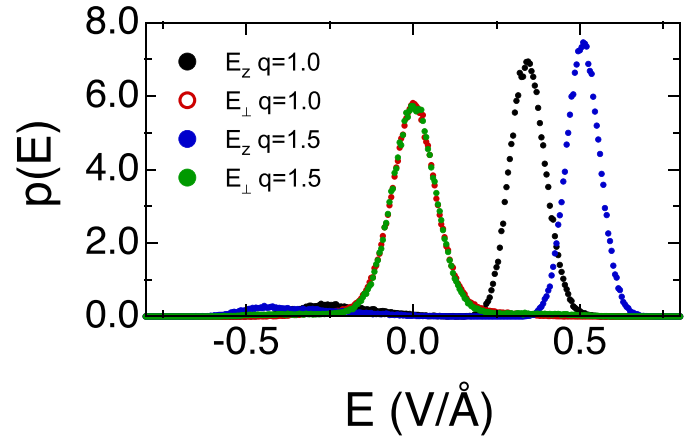

FIG. 21. Distribution $p\left(E_{s z}\right)$ of the Cartesian projection of $\mathbf{E}_{s}$ on the direction of the solute dipole and the mean distribution $\left[p\left(E_{s x}\right)+p\left(E_{s y}\right)\right] / 2$ of the perpendicular $\left(E_{\perp}\right)$ projections for the electric field created by SPC/E water at the geometrical center of the fused dumbbell solute (points). Calculations are done at $q=1.0$ and $1.5, T=300 \mathrm{~K}$.

mum of the stepwise probability distribution specified by the magnitude of the reaction field.

The dynamics of the electric field $\mathbf{E}_{s}$ was used to calculate $C_{E}(t)$ [Eq. (6)] and the integral relaxation time [Eq. (C12)]. The results for the integral relaxation time $\tau_{E}$ and electric field variance from MD simulations are summarized in Table I. It also lists the rotational relaxation time $\tau_{r}$ and the translational diffusion coefficient $D_{t}$ of the dumbbell solute. Both the increment of the solute rotational time $\Delta \tau_{r}$ over the hydrodynamic limit (Fig. 3) and $\tau_{E}$ (Fig. 6) follow approximately linear scalings with $m_{0}^{2}$. At large values of the solute dipole, we find $\Delta \tau_{r} \approx \tau_{E}$ as shown in Fig. 22.

\section{APPENDIX E: FORCES AND TORQUES}

The force acting on the solute from SPC/E water was divided into the electrostatic and $\mathrm{vdW}(\mathrm{LJ})$ components: $\mathbf{F}=\mathbf{F}_{E}+\mathbf{F}_{\mathrm{LJ}}$. The electrostatic force is given by the interaction energy of the water electric field with the solute charges $q_{i}, i=1,2$

$$
\mathbf{F}_{E}=\sum_{i=1,2} q_{i} \mathbf{E}_{s}(i)
$$

The electric field $\mathbf{E}_{s}(i)$ is calculated at each charge $q_{i}$, in contrast to the electric field $\mathbf{E}_{s}$ considered in Sec. D, which

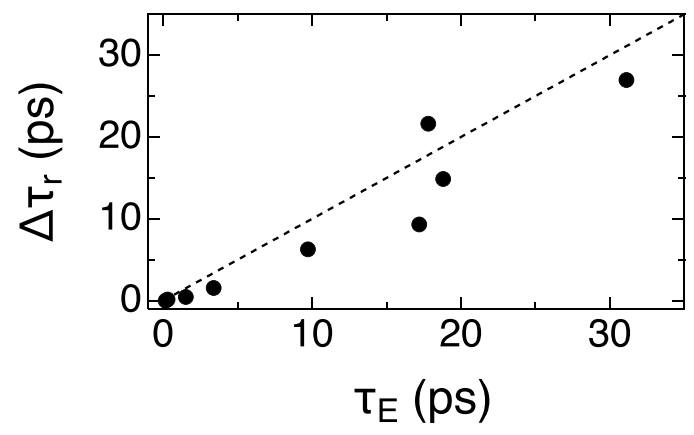

FIG. 22. $\Delta \tau_{r}$ vs $\tau_{E}$ calculated at different values of the solute dipole moment. The dashed line with the unit slope is drawn to indicate the equality between two values. 
TABLE II. Variance of the force acting on the dipolar solute from $\mathrm{SPC} / \mathrm{E}$ water at $T=300 \mathrm{~K}$. The variance is scaled with $\beta=\left(k_{B} T\right)^{-1}$ and is separated into the electric and LJ components and the crosscorrelation term $\left(\AA^{-2}\right)$.

\begin{tabular}{lcccc}
\hline \hline$q(\mathrm{e})$ & $\left\langle\left(\delta \mathbf{F}_{E}\right)^{2}\right\rangle$ & $\left\langle\left(\delta \mathbf{F}_{\mathrm{LJ}}\right)^{2}\right\rangle$ & $\left\langle(\delta \mathbf{F})^{2}\right\rangle$ & $-\left\langle\delta \mathbf{F}_{E} \cdot \delta \mathbf{F}_{\mathrm{LJ}}\right\rangle$ \\
\hline 0.05 & 0.35 & 71.6 & 71.0 & -0.43 \\
0.1 & 1.58 & 70.7 & 68.00 & -2.1 \\
0.4 & 10.2 & 110.8 & 94.0 & -13.5 \\
0.6 & 21.8 & 167.9 & 133.7 & -28.0 \\
0.9 & 59.3 & 345.7 & 257.0 & -74.0 \\
1.0 & 194.0 & 548.8 & 302.4 & -220.2 \\
1.3 & 413.2 & 1010.6 & 501.3 & -461.2 \\
1.4 & 544.8 & 1255.0 & 588.2 & -605.8 \\
1.5 & 818.6 & 1647.7 & 676.8 & -894.7 \\
\hline \hline
\end{tabular}

was calculated at the center of the solute. The $\mathrm{LJ}$ force $\mathbf{F}_{\mathrm{LJ}}$ is given as the gradient of the LJ interaction energy between the water molecules and two LJ sites within the solute (Fig. 18). Table II lists the components of the variance

$$
\left\langle(\delta \mathbf{F})^{2}\right\rangle=\left\langle\left(\delta \mathbf{F}_{E}\right)^{2}\right\rangle+\left\langle\left(\delta \mathbf{F}_{\mathrm{LJ}}\right)^{2}\right\rangle+2\left\langle\delta \mathbf{F}_{E} \cdot \delta \mathbf{F}_{\mathrm{LJ}}\right\rangle .
$$

Torques were calculated from the forces acting on the charges and $\mathrm{LJ}$ centers according to the standard equation

$$
\mathbf{T}=\sum_{i=1,2} \mathbf{s}_{i} \times \mathbf{f}_{i}
$$

where $\mathbf{f}_{i}$ are the forces acting on two interaction sites.

It is important to realize that when the distribution of forces acting on the solute $\mathbf{F}=\mathbf{F}_{\mathrm{LJ}}+\mathbf{F}_{E}$ is Gaussian and the distribution of the force magnitude $F$ is Maxwellian, the distribution of torques is not. This is easiest to show for the magnitude of the torque acting on a given site $T=T_{0} \sin \theta$, where $T_{0}=F s$ and $\theta$ is the angle between the force $\mathbf{f}_{i}$ acting on the site and $\mathbf{s}_{i}$. One obtains for the distribution of $T$ assuming that the force $F$ is distributed according to the three-dimensional (3D) Maxwell distribution

$$
p(T) \propto \int_{0}^{\infty} d T_{0} T_{0}^{2} \int_{0}^{\pi} d \theta \sin \theta \delta\left(T-T_{0} \sin \theta\right) \exp \left[-\frac{T_{0}^{2}}{2 \sigma^{2}}\right] .
$$

By integrating this equation, one obtains

$$
p(T, \sigma) \propto T e^{-T^{2} /\left(2 \sigma^{2}\right)} .
$$

The magnitude of the torque is therefore distributed according to the 2D Maxwell distribution. Figure 23 shows the results for the electrostatic and LJ torques indicating that this expectation is indeed realized.

To improve the quality of calculations of the torque variance from simulations, we fitted the distribution of torque magnitudes produced by MD to a sum of two 2D Maxwell distributions

$$
p(T)=\sum_{i=1,2} A_{i} p\left(T, \sigma_{i}\right), \quad \sum_{i=1,2} A_{i}=1 .
$$

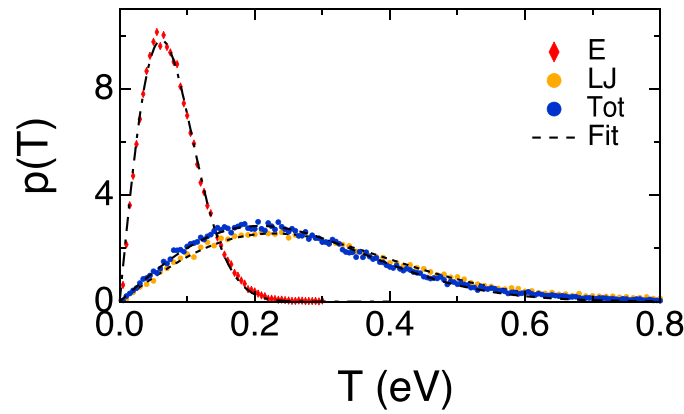

FIG. 23. Distribution of torques from electrostatic (E) and Lennard-Jones (LJ) interactions for $q=0.6$. Also shown is the distribution of the total torque (Tot); the dashed lines are fits to a single 2D Maxwell distribution [Eq. (E5)]. A linear combination of two 2D Maxwell distributions was used to calculate variances listed in Table III.

The variance was then calculated as

$$
\left\langle(\delta \mathbf{T})^{2}\right\rangle=\int_{0}^{\infty} d T T^{2} p(T)=2 \sum_{i=1,2} A_{i} \sigma_{i}^{2},
$$

where $\delta \mathbf{T}=\mathbf{T}-\langle\mathbf{T}\rangle=\mathbf{T}$ since $\langle\mathbf{T}\rangle=0$. The total variance of the torque is separated into the electrostatic and $\mathrm{vdW}(\mathrm{LJ})$ components and the cross term according to Eq. (18). These components, calculated from MD, are listed in Table III.

\section{APPENDIX F: SPC/E WATER}

MD simulations of SPC/E water [79] were carried out at a number of temperatures listed in Table IV. It presents the variance of the electric field at the water oxygen atom, the integral relaxation time of the field fluctuations $\tau_{E}$ [Eq. (C12)], and the variance of the torque applied to the center of mass including its vdW (LJ) and electrostatic components.

The variance of the electric field at the water oxygen is a linearly decaying function of temperature (Fig. 24). The relaxation time $\tau_{E}(T)$ is compared in Fig. 16 to the Debye relaxation time of SPC/E water calculated from MD and fitted to the Vogel-Fulcher equation [73]: $\tau_{D}(T)=\exp [-2.35+961 /$ $(T-88.2)]$. It is alternatively calculated from the continuum estimate in Eq. (12). The continuum $\tau_{E}^{c}$ is much smaller than

TABLE III. Components of the torque variance $\left(\mathrm{eV}^{2}\right)$ due to electrostatic (E) and vdW (LJ) interactions as listed in Eq. (18); $T=300 \mathrm{~K}$.

\begin{tabular}{lccccc}
\hline \hline$q(\mathrm{e})$ & $m_{0}(\mathrm{D})$ & $\left\langle\left(\delta \mathbf{T}_{E}\right)^{2}\right\rangle$ & $\left\langle\left(\delta \mathbf{T}_{\mathrm{LJ}}\right)^{2}\right\rangle$ & $\left\langle(\delta \mathbf{T})^{2}\right\rangle$ & $-\left\langle\delta \mathbf{T}_{E} \cdot \delta \mathbf{T}_{\mathrm{LJ}}\right\rangle$ \\
\hline 0.05 & 0.5 & 0.0004 & 0.038 & 0.032 & 0.003 \\
0.1 & 1.1 & 0.001 & 0.039 & 0.031 & 0.005 \\
0.4 & 4.2 & 0.005 & 0.083 & 0.056 & 0.016 \\
0.6 & 6.3 & 0.008 & 0.132 & 0.097 & 0.021 \\
0.9 & 9.5 & 0.015 & 0.264 & 0.201 & 0.039 \\
1.0 & 10.6 & 0.073 & 0.321 & 0.258 & 0.068 \\
1.3 & 13.7 & 0.077 & 0.528 & 0.434 & 0.086 \\
1.4 & 14.8 & 0.025 & 0.554 & 0.503 & 0.038 \\
1.5 & 15.9 & 0.257 & 0.861 & 0.592 & 0.263 \\
\hline \hline
\end{tabular}


TABLE IV. Properties of SPC/E water calculated from MD at different temperatures: Variance of the electric field $\left\langle\left(\delta \mathbf{E}_{s}\right)^{2}\right\rangle(\mathrm{V} / \AA)^{2}$, relaxation time $\tau_{E}(\mathrm{ps})$, and the components of the torque variance $\left(\mathrm{eV}^{2}\right)$.

\begin{tabular}{lccccc}
\hline \hline$T(\mathrm{~K})$ & $\left\langle\left(\delta \mathbf{E}_{s}\right)^{2}\right\rangle$ & $\tau_{E}$ & $\left\langle\left(\delta \mathbf{T}_{E}\right)^{2}\right\rangle$ & $\left\langle\left(\delta \mathbf{T}_{\mathrm{LJ}}\right)^{2}\right\rangle$ & $\left\langle(\delta \mathbf{T})^{2}\right\rangle$ \\
\hline 240 & 1.18 & 34.62 & 0.2587 & 0.0011 & 0.2580 \\
260 & 1.14 & 11.68 & 0.2597 & 0.0012 & 0.2591 \\
280 & 1.09 & 7.08 & 0.2608 & 0.0013 & 0.2605 \\
300 & 1.07 & 4.986 & 0.2658 & 0.0012 & 0.2656 \\
320 & 1.03 & 2.986 & 0.2787 & 0.0014 & 0.2789 \\
340 & 0.99 & 2.088 & 0.2851 & 0.0014 & 0.2860 \\
360 & 0.97 & 1.623 & 0.2779 & 0.0013 & 0.2842 \\
\hline
\end{tabular}

the MD result and $\tau_{E}(T)$ from MD falls slightly below the Debye relaxation time $\tau_{D}(T)$ (Fig. 16)

To evaluate the performance of the Nee-Zwanzig equation for SPC/E water, Fig. 25 shows the calculation of the second term in Eq. (4) in comparison to the rotational time of SPC/E water as tabulated in Ref. [67]. We find that Eq. (4) grossly overestimates the effect of dielectric friction on the rotational time (filled points in Fig. 25). On the other hand, electrostatic torque, which is dominating in the overall torque acting on the water molecule (Table IV), can be used to calculate the rotational time according to Eq. (21). These results are shown by the open points in Fig. 25. The agreement with direct MD calculations is better, but Eq. (21) still overestimates the rotational time. The reason is likely related to discontinuous jumps of water molecules speeding up rotations as compared to rotational diffusion [58].

\section{APPENDIX G: TRANSLATIONAL DIFFUSION AND SED VIOLATION}

The translational diffusion coefficients were calculated from the velocity autocorrelation functions as described in Sec. III and are listed in Table I. The diffusion coefficient in the body frame was calculated by rotating the solute velocity to the frame with the $z$ axis coinciding with the rotational symmetry axis of the solute (Fig. 18). Figure 26 shows the perpendicular, $D_{\perp}$, and parallel, $D_{\|}$, projections of the diffusion coefficient in the body frame plotted against the charge magnitude $q$. This figure shows that it is the parallel projection

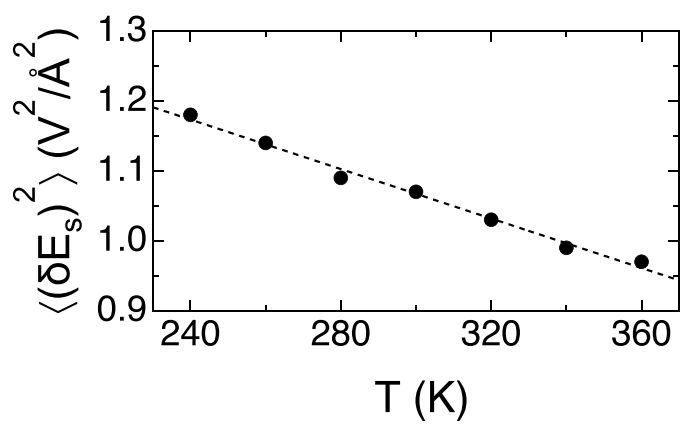

FIG. 24. Variance of the electric field at water's oxygen in bulk $\mathrm{SPC} / \mathrm{E}$ water calculated at different temperatures. The dashed line is a linear fit through the points.

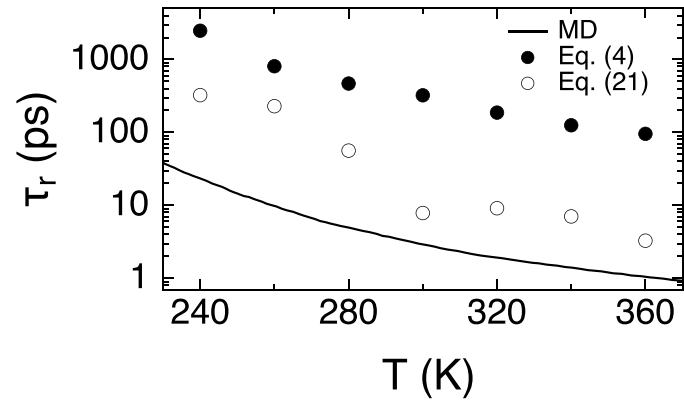

FIG. 25. $\tau_{r}$ from MD simulations of SPC/E water [67] (line, MD) compared to the second term in Eq. (4) calculated from our MD simulations (filled points, Table IV). Open points show the results of calculations by Eq. (21).

of the diffusion coefficient that is mostly affected by the solute dipole.

The results for SED violation are based on two sets of data. Figure 12 shows the product $D_{t} \tau_{r}$ vs the dipole moment of the solute, where $D_{t}$ is the translational diffusion coefficient in the laboratory frame. This plot is based on the data listed in Table I. In addition, Fig. 13 shows the dependence of $D_{t} \tau_{r}$ on $T^{-1}$ for the solute with the highest charge $\pm q$ placed at the spherical centers of the dumbbell solute, $q=1.5$. These data are listed in Table V.

\section{APPENDIX H: NON-GAUSSIAN PARAMETER}

From Eq. (32), one can calculate the fourth moment of $\Delta r_{x}(t)$

$$
\left\langle\Delta r_{x}(t)^{4}\right\rangle=\int_{0}^{t} d t_{1} \ldots d t_{4}\left\langle\xi_{x}\left(t_{1}\right) \ldots \xi_{x}\left(t_{4}\right)\right\rangle
$$

One gets

$$
\begin{aligned}
\left\langle\left[\Delta r_{x}(t)\right]^{4}\right\rangle= & 12 \bar{D}^{2}\left[t^{2}\left(1-\frac{\Delta D^{2}}{9 \bar{D}^{2}}\right)\right. \\
& \left.+\frac{\Delta D^{2}}{9 \bar{D}^{2}} \int_{0}^{t} \int_{0}^{t} d t_{1} d t_{2}\left\langle u_{x}\left(t_{1}\right)^{2} u_{x}\left(t_{2}\right)^{2}\right\rangle\right] .
\end{aligned}
$$

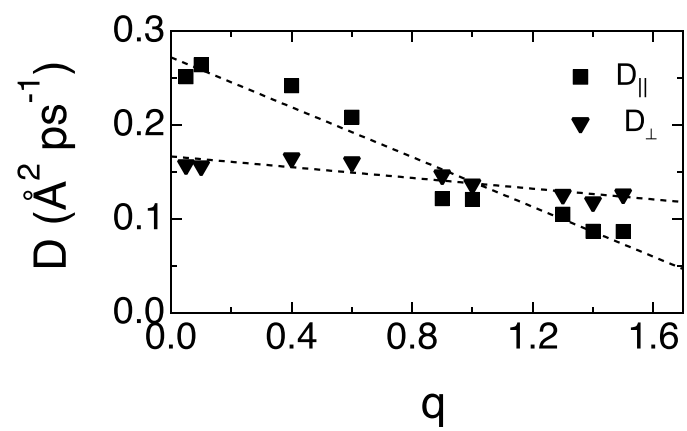

FIG. 26. Parallel, $D_{\|}$, and perpendicular, $D_{\perp}$, translational diffusion coefficients of the solute calculated in the body frame. The dashed lines are liner fits through the points. 
TABLE V. Diffusion coefficient $D_{t}\left(\AA^{2} / \mathrm{ps}\right)$ and the rotational time $\tau_{r}(\mathrm{ps})$ for the solute with $q=1.5$ at different temperatures.

\begin{tabular}{lcc}
\hline \hline$T(\mathrm{~K})$ & $D_{t}\left(\AA^{2} / \mathrm{ps}\right)$ & $\tau_{r}(\mathrm{ps})$ \\
\hline 245 & 0.0178 & 233.2 \\
252 & 0.0263 & 144.9 \\
260 & 0.0362 & 85.8 \\
267 & 0.0469 & 60.3 \\
275 & 0.0502 & 54.7 \\
290 & 0.0768 & 36.2 \\
300 & 0.0949 & 29.2 \\
\hline \hline
\end{tabular}

The average $\left\langle u_{x}\left(t_{1}\right)^{2} u_{x}\left(t_{2}\right)^{2}\right\rangle$ in Eq. (H2) is calculated from the propagator for rotational diffusion $P\left(\hat{\mathbf{u}}_{1}, t_{1} \mid \hat{\mathbf{u}}_{2}, t_{2}\right)$ [12]

$$
\left\langle u_{x}\left(t_{1}\right)^{2} u_{x}\left(t_{2}\right)^{2}\right\rangle=(4 \pi)^{-1} \int \hat{u}_{1 x}^{2} P\left(\hat{\mathbf{u}}_{1}, t_{1} \mid \hat{\mathbf{u}}_{2}, t_{2}\right) \hat{u}_{2 x}^{2} d \hat{\mathbf{u}}_{1} \hat{\mathbf{u}}_{2},
$$

where $t_{1}>t_{2}$ is assumed. The rotational propagator for a spherical particle with the rotational diffusion coefficient $D_{r}$ can be represented by an expansion in spherical harmonics $Y_{l m}\left(\hat{\mathbf{u}}_{i}\right)$ of the unit vectors $\hat{\mathbf{u}}_{i}$ specifying the solute orientation [12]

$$
P\left(\hat{\mathbf{u}}_{1}, t_{1} \mid \hat{\mathbf{u}}_{2}, t_{2}\right)=\sum_{l m} Y_{l m}\left(\hat{\mathbf{u}}_{1}\right) Y_{l m}^{*}\left(\hat{\mathbf{u}}_{2}\right) e^{-l(l+1) D_{r}\left|t_{1}-t_{2}\right|} .
$$

To perform integration over the orientations of the solute in Eq. (H3), one applies the expansion of the scalar product $\hat{u}_{x}=\hat{\mathbf{u}} \cdot \hat{\mathbf{x}}$ in spherical harmonics

$$
\hat{u}_{x}=\frac{4 \pi}{3} \sum_{m=-1}^{m=1} Y_{1 m}(\hat{\mathbf{u}}) Y_{1 m}^{*}(\hat{\mathbf{x}}) .
$$

The result of integration is

$$
\left\langle u_{x}\left(t_{1}\right)^{2} u_{x}\left(t_{2}\right)^{2}\right\rangle=\frac{1}{9}\left(1+e^{-D_{r}\left|t_{1}-t_{2}\right|}\right) .
$$

One therefore obtains

$$
\left\langle\left[\Delta r_{x}(t)\right]^{4}\right\rangle=12 \bar{D}^{2}\left[t^{2}+\frac{\Delta D^{2}}{9 \bar{D}^{2}} \int_{0}^{t} \int_{0}^{t} d t_{1} d t_{2} e^{-D_{r}\left|t_{1}-t_{2}\right|}\right] .
$$

and

$$
\left\langle\left[\Delta r_{x}(t)\right]^{4}\right\rangle-3\left\langle\left[\Delta r_{x}(t)\right]^{2}\right\rangle^{2}=\frac{4}{3} \Delta D^{2} \int_{0}^{t} \int_{0}^{t} d t_{1} d t_{2} e^{-D_{r}\left|t_{1}-t_{2}\right|} .
$$

By extending this result to three Cartesian components of the solute displacement, one gets

$$
\left\langle[\Delta \mathbf{r}(t)]^{4}\right\rangle-\frac{5}{3}\left\langle[\Delta \mathbf{r}(t)]^{2}\right\rangle^{2}=4 \Delta D^{2} \int_{0}^{t} \int_{0}^{t} d t_{1} d t_{2} e^{-D_{r}\left|t_{1}-t_{2}\right|} .
$$

Integration over time leads to the non-Gaussian parameter $\alpha_{2}(t)$ in Eq. (31)

$$
\alpha_{2}(t)=\frac{2 \Delta D^{2}}{15 \bar{D}^{2}} f\left(6 D_{r} t\right),
$$

where

$$
f(x)=\left(x-1+e^{-x}\right) / x^{2} .
$$

Equation (36) follows from Eq. (H10) by substituting $D_{r}=$ $\left(2 \tau_{r}\right)^{-1}$.
[1] A. Einstein, Investigations on the Theory of the Brownian Movement (BN Publishing, Hawthorne, CA, 2011).

[2] R. M. Mazo, Brownian Motion. Fluctuations, Dynamics, and Applications (Clarendon Press, Oxford, 2002).

[3] E. Frey and K. Kroy, Brownian motion: A paradigm of soft matter and biological physics, Ann. Phys. 14, 20 (2005).

[4] X. Bian, C. Kim, and G. E. Karniadakis, 111 years of Brownian motion, Soft Matter 12, 6331 (2016).

[5] J.-P. Hansen and I. R. McDonald, Theory of Simple Liquids, 4th ed. (Academic Press, Amsterdam, 2013).

[6] U. Balucani and M. Zoppi, Dynamics of the Liquid Phase (Clarendon Press, Oxford, 1994).

[7] R. Zwanzig, Time-correlation functions and transport coefficients in statistical mechanics, Annu. Rev. Phys. Chem. 16, 67 (1965).

[8] B. Bagchi, Molecular Relaxation in Liquids (Oxford University Press, Oxford, 2012).

[9] R. Zwanzig, Nonequilibrium Statistical Mechanics (Oxford University Press, Oxford, 2001).

[10] M. G. Mazza, N. Giovambattista, H. E. Stanley, and F. W. Starr, Connection of translational and rotational dynamical heterogeneities with the breakdown of the Stokes-Einstein and
Stokes-Einstein-Debye relations in water, Phys. Rev. E 76, 031203 (2007).

[11] T. Kawasaki and K. Kim, Spurious violation of the StokesEinstein-Debye relation in supercooled water, Sci. Rep. 9, 8118 (2019).

[12] B. J. Berne and R. Pecora, Dynamic Light Scattering (Dover, Mineola, NY, 2000).

[13] M. Born, Uber die Beweglichkeit det elektrolytischen Ionen, Z. Phys. 1, 221 (1920)

[14] T. W. Nee and R. R. Zwanzig, Theory of dielectric relaxation in polar liquids, J. Chem. Phys. 52, 6353 (1970).

[15] R. Zwanzig, Dielectric friction on a moving ion. II. Revised theory, J. Chem. Phys. 52, 3625 (1970).

[16] J. Hubbard and L. Onsager, Dielectric dispersion and dielectric friction in electrolyte solutions. I, J. Chem. Phys. 67, 4850 (1977).

[17] P. Wolynes, Dynamics of electrolyte solutions, Annu. Rev. Phys. Chem. 31, 345 (1980).

[18] R. Kubo, Some aspects of the statistical-mechanical theory of irreversible processes, in Lectures in Theoretical Physics (Interscience, New York, 1959), Vol. 1, p. 120. 
[19] T. Samanta and D. V. Matyushov, Mobility of large ions in water, J. Chem. Phys. 153, 044503 (2020).

[20] L. Onsager, Electric moments of molecules in liquids, J. Am. Chem. Soc. 58, 1486 (1936).

[21] M. Berkowitz and W. Wan, The limiting ionic conductivity of $\mathrm{Na}^{+}$and $\mathrm{Cl}^{-}$ions in aqueous solutions: Molecular dynamics simulation, J. Chem. Phys. 86, 376 (1987).

[22] S. Koneshan, J. C. Rasaiah, R. M. Lynden-Bell, and S. H. Lee, Solvent structure, dynamics, and ion mobility in aqueous solutions at $25^{\circ}$ C, J. Phys. Chem. B 102, 4193 (1998).

[23] S. H. Chong and F. Hirata, Dynamics of solvated ion in polar liquids: An interaction-site-model description, J. Chem. Phys. 108, 7339 (1998).

[24] J. C. Rasaiah and R. M. Lynden-Bell, Computer simulation studies of the structure and dynamics of ions and non-polar solutes in water, Philos. Trans. R. Soc. Lond. A 359, 1545 (2001)

[25] M. G. Kurnikova, N. Balabai, D. H. Waldeck, and R. D. Coalson, Rotational relaxation in polar solvents. Molecular dynamics study of solute-solvent interaction, J. Am. Chem. Soc. 120, 6121 (1998).

[26] P. V. Kumar and M. Maroncelli, The non-separability of "dielectric" and "mechanical" friction in molecular systems: A simulation study, J. Chem. Phys. 112, 5370 (2000).

[27] G. Tarjus and D. Kivelson, Breakdown of the Stokes-Einstein relation in supercooled liquids, J. Chem. Phys. 103, 3071 (1995).

[28] M. D. Ediger, Spatially heterogeneous dynamics in supercooled liquids, Annu. Rev. Phys. Chem. 51, 99 (2000).

[29] X. Xia and P. G. Wolynes, Diffusion and the mesoscopic hydrodynamics of supercooled liquids, J. Phys. Chem. B 105, 6570 (2001).

[30] R. Richert, Heterogeneous dynamics in liquids: Fluctuations in space and time, J. Phys.: Condens. Matter 14, R703 (2002).

[31] J. Qvist, C. Mattea, E. P. Sunde, and B. Halle, Rotational dynamics in supercooled water from nuclear spin relaxation and molecular simulations, J. Chem. Phys. 136, 204505 (2012).

[32] A. Dehaoui, B. Issenmann, and F. Caupin, Viscosity of deeply supercooled water and its coupling to molecular diffusion, Proc. Natl. Acad. Sci. USA 112, 12020 (2015).

[33] A. J. Cross and J. D. Simon, Rotational dynamics of a solvated dipole: A molecular dynamics study of dielectric friction, J. Chem. Phys. 86, 7079 (1987).

[34] M. Bruehl and J. T. Hynes, Dielectric friction and solvation dynamics: A molecular dynamics study, J. Phys. Chem. 96, 4068 (1992).

[35] J. B. Hubbard and P. G. Wolynes, Dielectric friction and molecular reorientation, J. Chem. Phys. 69, 998 (1978).

[36] R. S. Hartman, D. S. Alavi, and D. H. Waldeck, An experimental test of dielectric friction models using the rotational diffusion of aminoanthraquinones, J. Phys. Chem. 95, 7872 (1991).

[37] M. L. Horng, J. A. Gardecki, A. Papazyan, and M. Maroncelli, Subpicosecond measurements of polar solvation dynamics: Coumarin-153 revisited, J. Phys. Chem. 99, 17311 (1995).

[38] M. Maroncelli, The dynamics of solvation in polar liquids, J. Mol. Liq. 57, 1 (1993).

[39] G. van der Zwan and J. T. Hynes, Time-dependent fluorescence solvent shifts, dielectric friction, and nonequilibrium solvation in polar solvents, J. Phys. Chem. 89, 4181 (1985).
[40] E. W. Castner and M. Maroncelli, Solvent dynamics derived from optical Kerr effect, dielectric dispersion, and timeresolved stokes shift measurements: An empirical comparison, J. Mol. Liq. 77, 1 (1998).

[41] G. B. Dutt, G. R. Krishna, and S. Raman, Rotational dynamics of coumarins in nonassociative solvents: Point dipole versus extended charge distribution models of dielectric friction, J. Chem. Phys. 115, 4732 (2001).

[42] B. R. Gayathri, J. R. Mannekutla, and S. R. Inamdar, Rotational diffusion of coumarins in alcohols: A dielectric friction study, J. Fluoresc. 18, 943 (2008).

[43] S. Riniker, A.-P. E. Kunz, and W. F. van Gunsteren, On the calculation of the dielectric permittivity and relaxation of molecular models in the liquid phase, J. Chem. Theory Comput. 7, 1469 (2011).

[44] H. S. Frank and W.-Y. Wen, Ion-solvent interaction. Structural aspects of ion-solvent interaction in aqueous solutions: A suggested picture of water structure, Discuss. Faraday Soc. 24, 133 (1957).

[45] S. Mondal, S. Acharya, and B. Bagchi, Altered polar character of nanoconfined liquid water, Phys. Rev. Research 1, 033145 (2019).

[46] S. Prager, Interaction of rotational and translational diffusion, J. Chem. Phys. 23, 2404 (1955).

[47] Y. Han, A. M. Alsayed, M. Nobili, J. Zhang, T. C. Lubensky, and A. G. Yodh, Brownian motion of an ellipsoid, Science 314, 626 (2006).

[48] M. Molaei, E. Atefi, and J. C. Crocker, Nanoscale Rheology and Anisotropic Diffusion Using Single Gold Nanorod Probes, Phys. Rev. Lett. 120, 118002 (2018).

[49] J. K. G. Dhont, An Introduction to Dynamics of Colloids, Studies in Interface Science Vol. 2 (Elsevier Science, Amsterdam, 1996).

[50] H. Goldstein, Classical Mechanics (Addison-Wesley, Reading, MA, 1964).

[51] A. Rahman, Correlations in the motion of atoms in liquid argon, Phys. Rev. 136, A405 (1964).

[52] W. Kob, C. Donati, S. J. Plimpton, P. H. Poole, and S. C. Glotzer, Dynamical Heterogeneities in a Supercooled LennardJones Liquid, Phys. Rev. Lett. 79, 2827 (1997).

[53] L. Berthier and G. Biroli, Theoretical perspective on the glass transition and amorphous materials, Rev. Mod. Phys. 83, 587 (2011).

[54] A. D. S. Parmar, S. Sengupta, and S. Sastry, Length-Scale Dependence of the Stokes-Einstein and Adam-Gibbs Relations in Model Glass Formers, Phys. Rev. Lett. 119, 056001 (2017).

[55] D. Paschek and A. Geiger, Simulation study on the diffusive motion in deeply supercooled water, J. Phys. Chem. B 103, 4139 (1999).

[56] C. A. Angell, Formation of glasses from liquids and biopolymers, Science 267, 1924 (1995).

[57] I. Chang and B. Sillescu, Heterogeneity at the glass transition: Translational and rotational self-diffusion, J. Phys. Chem. 101, 8794 (1997).

[58] D. Laage and J. T. Hynes, A molecular jump mechanism of water reorientation, Science 311, 832 (2006).

[59] N. Galamba, On the hydrogen-bond network and the nonArrhenius transport properties of water, J. Phys.: Condens. Matter 29, 015101 (2017). 
[60] C. J. F. Böttcher, Theory of Electric Polarization, Vol. 1: Dielectrics in Static Fields (Elsevier, Amsterdam, 1973).

[61] H. J. Bakker and J. L. Skinner, Vibrational spectroscopy as a probe of structure and dynamics in liquid water, Chem. Rev. 110, 1498 (2010).

[62] D. V. Matyushov and R. Richert, Communication: Temperature derivative of the dielectric constant gives access to multipoint correlations in polar liquids, J. Chem. Phys. 144, 041102 (2016).

[63] H. Tanaka, Simple physical model of liquid water, J. Chem. Phys. 112, 799 (2000).

[64] L. P. Singh, B. Issenmann, and F. Caupin, Pressure dependence of viscosity in supercooled water and a unified approach for thermodynamic and dynamic anomalies of water, Proc. Natl. Acad. Sci. USA 114, 4312 (2017).

[65] P. M. de Hijes, E. Sanz, L. Joly, C. Valeriani, and F. Caupin, Viscosity and self-diffusion of supercooled and stretched water from molecular dynamics simulations, J. Chem. Phys. 149, 094503 (2018).

[66] D. Rozmanov and P. G. Kusalik, Transport coefficients of the TIP4P-2005 water model, J. Chem. Phys. 136, 044507 (2012).

[67] N. Meyer, V. Piquet, J. F. Wax, H. Xu, and C. Millot, Rotational and translational dynamics of the SPC/E water model, J. Mol. Liq. 275, 895 (2019).

[68] P. K. Ghorai and D. V. Matyushov, Solvent reorganization of electronic transitions in viscous solvents, J. Chem. Phys. 124, 144510 (2006).

[69] T. Kawasaki and K. Kim, Identifying time scales for violation/preservation of Stokes-Einstein relation in supercooled water, Sci. Adv. 3, e1700399 (2017).

[70] E. A. Carter and J. T. Hynes, Solvation dynamics for an ion pair in a polar solvent: Time-dependent fluorescence and photochemical charge transfer, J. Chem. Phys. 94, 5961 (1991).

[71] T. Fonseca and B. M. Ladanyi, Breakdown of linear response for solvation dynamics in methanol, J. Phys. Chem. 95, 2116 (1991).
[72] D. R. Martin and D. V. Matyushov, Why are vibrational lines narrow in proteins? J. Phys. Chem. Lett. 11, 5932 (2020).

[73] P. K. Ghorai and D. V. Matyushov, Solvent reorganization energy in solvents above the glass transition, J. Phys. Chem. B 110, 1866 (2006).

[74] C. G. Gray and K. E. Gubbins, Theory of Molecular Liquids, Vol. 1: Fundamentals (Clarendon Press, Oxford, 1984).

[75] S. Takashima, Electric dipole moment of globular proteins: Measurement and calculation with NMR and X-ray databases, J. Noncrystal. Solids 305, 303 (2002).

[76] S. M. Sarhangi and D. V. Matyushov, Driving forces of protein diffusivity, J. Phys. Chem. Lett. 11, 10137 (2020).

[77] H. Sillescu, Heterogeneity at the glass transition: A review, J. Non-Cryst. Solids 243, 81 (1999).

[78] R. Zwanzig, Rate processes with dynamical disorder, Acc. Chem. Res. 23, 148 (1990).

[79] H. J. C. Berendsen, J. R. Grigera, and T. P. Straatsma, The missing term in effective pair potentials, J. Phys. Chem. 91, 6269 (1987).

[80] M. P. Allen and D. J. Tildesley, Computer Simulation of Liquids (Clarendon Press, Oxford, 1996).

[81] D. V. Matyushov, Solvent reorganization energy of electron transfer in polar solvents, J. Chem. Phys. 120, 7532 (2004).

[82] D. Chandler, Gaussian field model of fluids with an application to polymeric fluids, Phys. Rev. E 48, 2898 (1993).

[83] D. V. Matyushov, Dipole solvation in dielectrics, J. Chem. Phys. 120, 1375 (2004).

[84] Handbook of Mathematical Functions, edited by $\mathrm{M}$ Abramowitz and I. A. Stegun (Dover, New York, 1972).

[85] S. Seyedi, D. R. Martin, and D. V. Matyushov, Screening of Coulomb interactions in liquid dielectrics, J. Phys.: Condens. Matter 31, 325101 (2019).

[86] L. D. Landau and E. M. Lifshitz, Mechanics, 3rd ed., Course of Theoretical Physics Vol. 1 (Elsevier, Amsterdam, 2007). 